\title{
WINDOWED GREEN FUNCTION METHOD FOR LAYERED-MEDIA SCATTERING*
}

\author{
OSCAR P. BRUNO ${ }^{\dagger}$, MARK LYON $^{\ddagger}$, CARLOS PÉREZ-ARANCIBIA $^{\dagger}$, AND \\ CATALIN TURC $\$$
}

\begin{abstract}
This paper introduces a new windowed Green function (WGF) method for the numerical integral-equation solution of problems of electromagnetic scattering by obstacles in the presence of dielectric or conducting half-planes. The WGF method, which is based on the use of smooth windowing functions and integral kernels that can be expressed directly in terms of the free-space Green function, does not require evaluation of expensive Sommerfeld integrals. The proposed approach is fast, accurate, flexible, and easy to implement. In particular, straightforward modifications of existing (accelerated or unaccelerated) integral-equation solvers suffice to incorporate the WGF capability. The method relies on a certain integral equation posed on the union of the boundary of the obstacle and a small flat section of the interface between the penetrable media. Our analysis and numerical experiments demonstrate that both the near- and far-field errors resulting from the proposed approach decrease faster than any negative power of the window size. In the examples considered in this paper the proposed method is up to thousands of times faster, for a given accuracy, than a corresponding method based on use of Sommerfeld integrals.
\end{abstract}

Key words. Sommerfeld integrals, layered-media scattering, layer Green function

AMS subject classifications. 15A15, 15A09, 15A23

DOI. $10.1137 / 15 \mathrm{M} 1033782$

1. Introduction. The problem of scattering in the presence of layer media plays important roles in a wide range of contexts, with application in simulations of printed circuits, patch antennas, ground-plane cloaks, multilayer aircraft coatings (for stealth applications), partially or completely buried objects, well logging, propagation of radio waves on the earth surface and ionosphere, etc. The solution of problems of scattering by obstacles or defects in the presence of planar layered dielectric or conducting media has typically required the use of Sommerfeld integrals and associated layer Green functions - which automatically enforce the relevant transmission conditions on the unbounded flat surfaces and thus reduce the scattering problems to integral equations on the obstacles and/or defects. As is well known, however, the numerical evaluation of layer Green functions and their derivatives, which amounts to computation of certain challenging Fourier integrals $[9,28]$, is extremely expensive and gives rise to a significant bottleneck in layer-media simulations (see, e.g., [7] for details). This paper presents a novel integral-equation approach for problems involving layered media. The new approach, which is based on use of certain "windowing" functions and considerations associated with the method of stationary phase, does

*Received by the editors August 4, 2015; accepted for publication (in revised form) July 7, 2016; published electronically September $22,2016$.

http://www.siam.org/journals/siap/76-5/M103378.html

Funding: The first author's research was supported by the NSF and AFOSR through contracts DMS-1411876 and FA9550-15-1-0043. The fourth author's research was supported by the NSF through contract DMS-1312169.

$\dagger$ Department of Computing \& Mathematical Sciences, California Institute of Technology, Pasadena, CA 91125 (obruno@caltech.edu, cperezar@caltech.edu).

${ }^{\ddagger}$ Department of Mathematics and Statistics, University of New Hampshire, Durham, NH 03824 (mark.lyon@unh.edu).

$\S$ Department of Mathematics, New Jersey Institute of Technology, Newark, NJ 07102 (catalin.c. turc@njit.edu). 
not require the use of expensive Sommerfeld integrals. Our analysis and numerical experiments demonstrate that both the near- and far-field errors resulting from the proposed approach decrease faster than any negative power of the window size.

A variety of methods have been provided for the solution of problems of scattering by obstacles in the presence of layered media. Amongst the most effective such approaches we mention (1) methods which evaluate Sommerfeld integrals on the basis of path-integration in the complex plane $[7,8,25,26]$ (such approaches require numerical evaluation of integrals of functions that oscillate, grow exponentially in a bounded section of the integration path, and, depending on the relative position of the source and observation points to the interface between the two media, may decay slowly at infinity; (2) the complex images method reviewed in [1] (a discussion indicating certain instabilities and inefficiencies in this method is presented in [8, section 5.5]); (3) the steepest descent method $[12,13]$, which, provided the steepest descent path is known, reduces the Sommerfeld integral to an integral of an exponentially decaying function (unfortunately, however, the application of the steepest descent method for each observation point can be challenging and expensive $[7,8,12])$; (4) the contribution [20], which, utilizing Laplace transforms in addition to the Fourier transforms in the Sommerfeld method, demonstrates an improved performance over direct integration of the Sommerfeld integrals - but, as the authors stress, this is probably due to the straightforward character of the Sommerfeld integration method they use; and (5) a method presented in [24] that relies on a combination of Sommerfeld integral representations as well as the method of images for a related application to the impedance problem, and which is demonstrated in low-frequency contexts. As is known, in any case, all of these methods entail significant computational costs [7, 20,24].

The ideas embodied in the windowed Green function (WGF) method proposed in this paper are related to apodization techniques used in optics, as well as tapering or Hann functions utilized widely in signal processing. Apodization is used in the design of certain optical devices to eliminate edge effects; the Hann functions, in turn, are used to produce signals of finite duration from infinite-time signals while reducing distortions in the spectrum caused by the windowing process itself. From a computational perspective in a problem related to wave scattering, finally, the approach proposed in this paper bears similarities with certain "finite-section" methods in the field of rough-surface scattering. These methods utilize approximations based on truncated portions of a given unbounded rough surface [21, 27, 30], and, in some cases, they incorporate a "taper" $[22,29,30]$ to eliminate artificial reflections from the edges of the finite sections. In fact the smooth taper function utilized in [22] (Figure 2 in that reference) resembles the smooth windowing function we use (Figure 3.1 below and $[5,23]$ ). But as indicated in comments provided in section 3.1 in regards to certain slow-rise windowing functions, essential differences exist between the finite-section approaches and the methods proposed in this paper. In particular, with exception of the slow-rise windowing function method $[5,23]$, none of the previous tapered rough surface algorithms has demonstrated high-order convergence as the width of the finite sections tend to infinity.

In section 5 the proposed WGF method is compared to the high-order integral equation method recently introduced in [26], which is based on the accurate and efficient evaluation of Sommerfeld integrals. In the examples considered in that section the proposed method is up to thousands of times faster, for a given accuracy, than the corresponding layer-Green-function method. A similar improvement in computational costs has been observed for problems of electromagnetic scattering by defects and obstacles in multilayer structures in two and three dimensions; a detailed discussion 
of such problems, however, is left for future work.

This paper is organized as follows. After some basic preliminaries are presented in section 2, the proposed methodology is introduced in section 3. A formal error analysis of the method, based on multiple-scattering perturbation theory, then follows in section 4 . A variety of numerical results presented in section 5 , finally, demonstrate the accuracy and speed of the proposed approach.

2. Preliminaries. We consider two-dimensional problems of reflection and transmission by dielectric or conducting media under TE and TM polarizations. As is well known, the $z$ components $u=E_{z}$ and $u=H_{z}$ of the total electric and magnetic fields satisfy the Helmholtz equation $\Delta u+k_{j}^{2} u=0$ in $\Omega_{j}, j=1,2$ (see Figure 2.1), where calling $\mu_{0}>0$ and $\omega>0$ the magnetic permeability of vacuum and the temporal frequency, and letting $\varepsilon_{j}>0$ and $\sigma_{j} \geq 0\left(\sigma_{1}=0\right)$ denote the electric permittivity and the electrical conductivity in $\Omega_{j}$, the corresponding wavenumbers $k_{j}$ are defined by $k_{j}^{2}=\omega^{2}\left(\varepsilon_{j}+i \sigma_{j} / \omega\right) \mu_{0}, j=1,2$. In either case the total field resulting from a plane-wave incident field

$$
u^{\text {inc }}(\boldsymbol{x})=\mathrm{e}^{i k_{1}\left(x_{1} \cos \alpha+x_{2} \sin \alpha\right)}
$$

with incidence angle $\alpha \in(-\pi, 0)$ measured from the horizontal (see Figure 2.1) is given by

$$
u=\left\{\begin{array}{ccc}
u_{1}+u^{\text {inc }} & \text { in } & \Omega_{1}, \\
u_{2} & \text { in } & \Omega_{2},
\end{array}\right.
$$

where $u_{1}$ and $u_{2}$ denote the reflected and transmitted waves, respectively. As is known (see, e.g., [15, section 5]), the scattered and transmitted fields $u_{1}$ and $u_{2}$ admit the representations

$$
\begin{aligned}
& u_{1}=\mathscr{D}_{1}\left[\left.u_{1}\right|_{\Gamma}\right]-\mathscr{S}_{1}\left[\left.\frac{\partial u_{1}}{\partial n}\right|_{\Gamma}\right] \quad \text { in } \Omega_{1}, \\
& u_{2}=-\mathscr{D}_{2}\left[\left.u_{2}\right|_{\Gamma}\right]+\mathscr{S}_{2}\left[\left.\frac{\partial u_{2}}{\partial n}\right|_{\Gamma}\right]+u^{\|} \text {in } \Omega_{2},
\end{aligned}
$$

where

$$
u^{\|}(\boldsymbol{x})=\left\{\begin{array}{ccc}
\mathrm{e}^{i k_{1} x_{1} \cos \alpha} & \text { if } & k_{2}=k_{1}|\cos \alpha|, \\
0 & \text { if } & k_{2} \neq k_{1}|\cos \alpha|,
\end{array}\right.
$$

and where, letting

$$
G_{j}(\boldsymbol{x}, \boldsymbol{y})=\frac{i}{4} H_{0}^{(1)}\left(k_{j}|\boldsymbol{x}-\boldsymbol{y}|\right), \quad j=1,2,
$$

denote the free-space Green function for the Helmholtz equation with wavenumber $k_{j}$, the single- and double-layer potentials in (2.3) are defined by means of the improper integrals

$$
\mathscr{S}_{j}[\eta](\boldsymbol{x})=\int_{\Gamma} G_{j}(\boldsymbol{x}, \boldsymbol{y}) \eta(\boldsymbol{y}) \mathrm{d} s_{\boldsymbol{y}} \quad \text { and } \quad \mathscr{D}_{j}[\eta](\boldsymbol{x})=\int_{\Gamma} \frac{\partial G_{j}}{\partial n_{\boldsymbol{y}}}(\boldsymbol{x}, \boldsymbol{y}) \eta(\boldsymbol{y}) \mathrm{d} s_{\boldsymbol{y}},
$$

respectively - whose convergence is conditioned upon the oscillatory behavior of the integrand. Throughout this paper the interface $\Gamma$ is assumed to be a piecewise smooth 


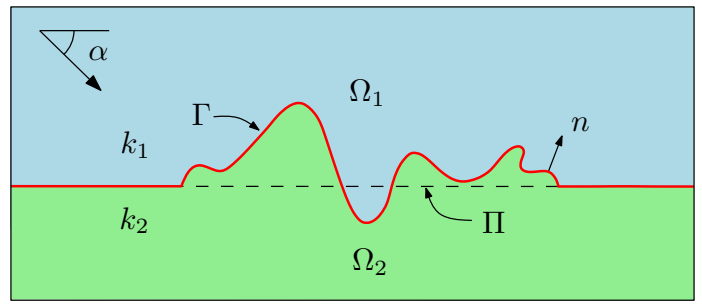

FIG. 2.1. Description of the problem under consideration: scattering by a defect on a penetrable planar dielectric or conducting layer. $\Gamma$ denotes the interface between the two media, and $\Pi$ denotes the interface between the upper and lower half-planes.

curve that coincides with the flat interface $\left\{x_{2}=0\right\}$ for large enough values of $\left|x_{1}\right|$; see, e.g., Figure 2.1.

By evaluating the fields (2.3) and their normal derivatives on $\Gamma$ and using the transmission conditions

$$
u_{2}-u_{1}=u^{\mathrm{inc}}, \quad \nu \frac{\partial u_{2}}{\partial n}-\frac{\partial u_{1}}{\partial n}=\frac{\partial u^{\mathrm{inc}}}{\partial n} \quad \text { on } \quad \Gamma
$$

(with $\nu=1$ and $\nu=\varepsilon_{1} / \varepsilon_{2}$ in TE- and TM-polarizations, respectively), we obtain the second-kind system of integral equations [17]

$$
E \phi+T[\phi]=\phi^{\text {inc }} \quad \text { on } \quad \Gamma
$$

for the surface currents $\phi$. Here

$$
E=\left[\begin{array}{cc}
1 & 0 \\
0 & \frac{1+\nu}{2}
\end{array}\right], \quad \phi=\left[\begin{array}{c}
\left.u_{2}\right|_{\Gamma} \\
\left.\frac{\partial u_{2}}{\partial n}\right|_{\Gamma}
\end{array}\right], \quad \phi^{\text {inc }}=\left[\begin{array}{c}
\left.\left(u^{\mathrm{inc}}+u^{\|}\right)\right|_{\Gamma} \\
\left.\frac{\partial\left(u^{\mathrm{inc}}+u^{\|}\right)}{\partial n}\right|_{\Gamma}
\end{array}\right]
$$

and

$$
T=\left[\begin{array}{cc}
D_{2}-D_{1} & -S_{2}+\nu S_{1} \\
N_{2}-N_{1} & -K_{2}+\nu K_{1}
\end{array}\right]
$$

where, using the potentials (2.5), the entries in the matrix operator $T$ are defined by

$$
\begin{aligned}
S_{j}[\eta](\boldsymbol{x}) & =\mathscr{S}_{j}[\eta](\boldsymbol{x}), & D_{j}[\eta](\boldsymbol{x}) & =\int_{\Gamma} \frac{\partial G_{j}}{\partial n_{\boldsymbol{y}}}(\boldsymbol{x}, \boldsymbol{y}) \eta(\boldsymbol{y}) \mathrm{d} s_{\boldsymbol{y}}, \\
N_{j}[\eta](\boldsymbol{x}) & =\frac{\partial \mathscr{D}_{j}[\eta]}{\partial \eta}(\boldsymbol{x}), & K_{j}[\eta](\boldsymbol{x}) & =\int_{\Gamma} \frac{\partial G_{j}}{\partial n_{\boldsymbol{x}}}(\boldsymbol{x}, \boldsymbol{y}) \eta(\boldsymbol{y}) \mathrm{d} s_{\boldsymbol{y}}
\end{aligned}
$$

for $\boldsymbol{x} \in \Gamma$ and for $j=1,2$.

\section{WGF method: Basic concepts.}

3.1. Slow-rise windowing function. Instead of solving the problem (2.6) on the entire infinite interface $\Gamma$, a locally windowed problem could be used in an attempt to obtain the local currents over all relevant portions of $\Gamma$ in an inexpensive manner. To pursue this idea we introduce a smooth windowing function $w_{A}$ depicted in Figure 3.1, which is nonzero in an interval of length $2 A$, and which has a slow rise - that is,

$$
w_{A}\left(x_{1}\right)=w\left(x_{1} / A ; c, 1\right)
$$

Copyright (c) by SIAM. Unauthorized reproduction of this article is prohibited. 
for some fixed window function $w$. Throughout this paper the window function

$$
w\left(s ; s_{0}, s_{1}\right)=\left\{\begin{array}{cl}
1, & |s| \leq s_{0}, \\
\exp \left(\frac{2 \mathrm{e}^{-1 / u}}{u-1}\right), & s_{0}<|s|<s_{1}, u=\frac{|s|-s_{0}}{s_{1}-s_{0}}, \\
0, & |s|>s_{1},
\end{array}\right.
$$

is used. (The value $c=0.7$ was utilized to produce all of the numerical examples presented in this paper.) Clearly, $w_{A}$ rises from zero to one in regions of length proportional to $A>0$. As demonstrated in $[5,23]$ the slow-rise character of the window function $w_{A}$ is essential to ensure superalgebraically fast convergence (i.e., faster than any power of $1 / A$ ) of windowed oscillatory integrals. Throughout this paper the function $w_{A}$, which only depends on $x_{1}$, is viewed as a function defined for all values of $\left(x_{1}, x_{2}\right) \in \mathbb{R}^{2}$ - which is constant with respect to $x_{2}$ for each fixed value of $x_{1}$.

The parts of the boundary $\Gamma$ where $w_{A}\left(x_{1}\right) \neq 0$ and $\widetilde{w}_{A}\left(x_{1}\right)=1-w_{A}\left(x_{1}\right) \neq 0$ will be denoted by $\Gamma_{A}$ and $\widetilde{\Gamma}_{A}$, respectively. The width $2 A>0$ is only restricted by the requirement that $\widetilde{w}_{A}\left(x_{1}\right)$ vanishes on any corrugations that exist on the surface $\Gamma$ as well as on any additional obstacles that may exist above and/or below $\Gamma$. As shown below in this text, solutions converge rapidly as $A$ increases beyond the bound posed by this restriction.

Remark 3.1. For notational simplicity our derivations in the remainder of section 3 are presented for cases for which the corrugations on the surface $\Gamma$ are the only departures from planarity (see, e.g., Figure 2.1). Cases in which additional scatterers exist (e.g., Figures 4.5 and 5.3) are considered in sections 4 and 5.

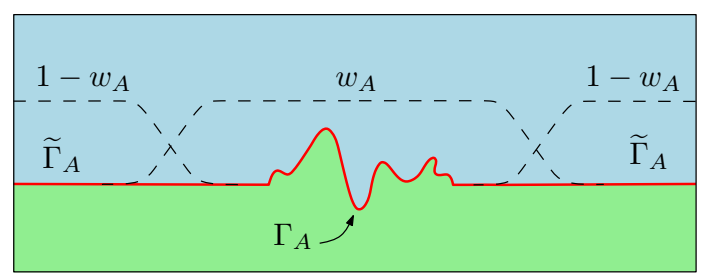

FIG. 3.1. Window function $w_{A}$ and the windowed sections $\Gamma_{A}$ and $\widetilde{\Gamma}_{A}$ of the unbounded curve $\Gamma$.

3.2. Windowed integral equation: Preliminary considerations. With reference to Remark 3.1, letting $W_{A}=w_{A} \cdot I$, where $I$ is the $2 \times 2$ identity matrix, we consider the preliminary approximate equation

$$
E \phi^{\star}+T\left[W_{A} \phi^{\star}\right]=\phi^{\text {inc }} \quad \text { on } \Gamma_{A},
$$

where $\phi^{\star}$ denotes a new unknown defined on $\Gamma_{A}$. In order to assess the errors inherent in this approximation, we also consider the form

$$
E \phi+T\left[W_{A} \phi\right]=\phi^{\text {inc }}-T\left[\left(I-W_{A}\right) \phi\right] \quad \text { on } \quad \Gamma_{A}
$$

of the exact equation (2.6).

As indicated in section 4, arguments based on integration by parts and the concept of stationary-phase can be used to establish that both the right-hand side 
$T\left[\left(I-W_{A}\right) \phi\right]$ in (3.3) and the approximation error $\left|\phi-\boldsymbol{\phi}^{\star}\right|$ are superalgebraically small-i.e., smaller than $C A^{-m}$ for any positive integer $m$ as $A \rightarrow \infty$, where the constant $C$ is independent of $A$-throughout the center region $\left\{w_{A}=1\right\}$ of the surface $\Gamma_{A}$. However, large window sizes may be required in such a scheme to correctly account for all fields reflected and refracted by the planar surface - a difficulty that can be visualized easily for incidence angles approaching grazing.

In order to demonstrate this difficulty (which is in fact overcome in section 3.4 by incorporating certain closed-form and numerically evaluated expressions), here we consider a test case in which (3.2) is used to approximate the solution of the TEproblem of scattering of a plane-wave by a semicircular bump of radius $a=1$ placed directly on top of a planar dielectric surface (see, e.g., Figure 3.5). The problem was discretized using a direct generalization of the Nyström method presented in [18] which, relying on graded meshes over the surfaces of the bump and the windowed portion of the planar interface, accurately accounts for the singularities of the currents at and around corners. For this example the wavenumbers $k_{1}$ and $k_{2}$ in the regions above and below the plane were set to $4 \pi$ and $8 \pi$, respectively, and approximately 20 points per unit length of the surface of the bump and the surrounding were used.

Figure 3.2 shows that, as suggested above, the naive windowing approach embodied in (3.2) requires, for a given accuracy, large values of $A$-well beyond the extent of the nonplanar local geometry - as the incidence angle decreases. A correction that resolves this difficulty, and which results in superalgebraic convergence uniformly for all incidence angles, is presented in section 3.4.
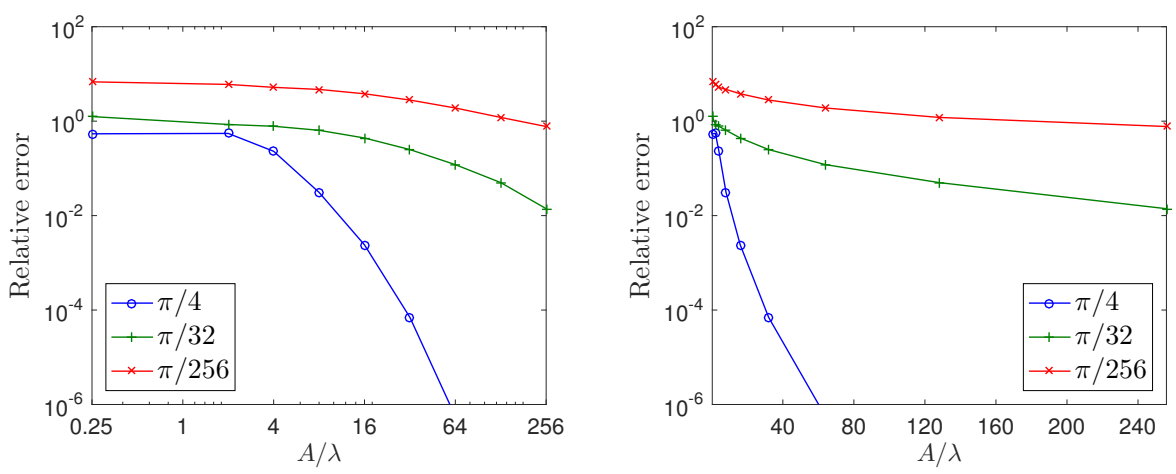

FIG. 3.2. Relative errors (see Remark 5.1) in the integral densities resulting from numerical solution of (3.2) by means of a naive implementation of the WGF method for a semicircular bumpshaped defect, for various window sizes (measured in numbers $A / \lambda$ of wavelengths, where $\lambda=2 \pi / k_{1}$ denotes the free-space wavelength) and angles of incidence $\alpha=-\pi / 4$ (blue), $-\pi / 32$ (green), and $-\pi / 256$ (red). Left: log-log scale. Right: semi-log scale. Clearly, the window size required by the naive method to produce a given accuracy increases dramatically as the angle of incidence approaches grazing. Color is available online only.

3.3. Error sources in (3.2). In order to provide an insight into the source of the errors displayed in Figure 3.2, we present Figure 3.3. Figure 3.3(a) depicts rays incident on the left planar region as well as their reflection and transmission. Clearly, in view of the incidence angle considered in this example, these reflected fields subsequently illuminate the defect and thus give rise to multiple scattering.

Remark 3.2. In the present section we make free use of standard geometrical optics nomenclature, with mention, in particular, of shooting and bouncing rays. Addi- 
tionally, we make reference to the property of superalgebraic convergence that arises from the windowing of integral representations of scattered fields around points of stationary-phase [6]. A justification of the geometrical-optics and integral-asymptotics arguments used throughout section 3 is provided in section 4 - on the basis of the concept of stationary phase, and the methods of contour integration and multiplescattering perturbation theory.

Continuing with our argument concerning Figure 3.3(a), then, let us consider separately the rays shown in blue and red (or, in gray-scale, dark gray and light gray, respectively) in that figure. The blue rays represent the reflections that are correctly taken into account in the solution of (3.2) (since they impinge within the windowed region), but, clearly, the red arrows represent reflections that are neglected in this equation. Figure 3.3(b), on the other hand, represents reflections by the defect. The color-code in the left figure carries over to the right figure: the blue (resp., red) rays in Figure 3.3(b) represent the fields scattered by the defect which arise from the blue (resp., red) arrows in Figure 3.3(a). It is natural to suggest that, as justified in section 4 , the omission of the incident fields represented by the red arrows causes the errors observed in Figure 3.2. We also note that the relatively fast convergence demonstrated by the blue curves in Figure 3.2 can be explained by the fact that for near normal incidence $(\alpha \approx-\pi / 2)$ there is not a significant "red field" interacting with the defect. In contrast, for incidence near grazing $(\alpha \approx 0)$, "red fields" from regions far away from the windowed area do interact with the defect, and therefore give rise to significant errors if neglected. As shown in section 3.4, the introduction of adequate corrections in (3.2) which account for such neglected terms allows us to establish superalgebraically fast convergence uniformly over the domain $[-\pi, 0]$ of all possible incidence angles.
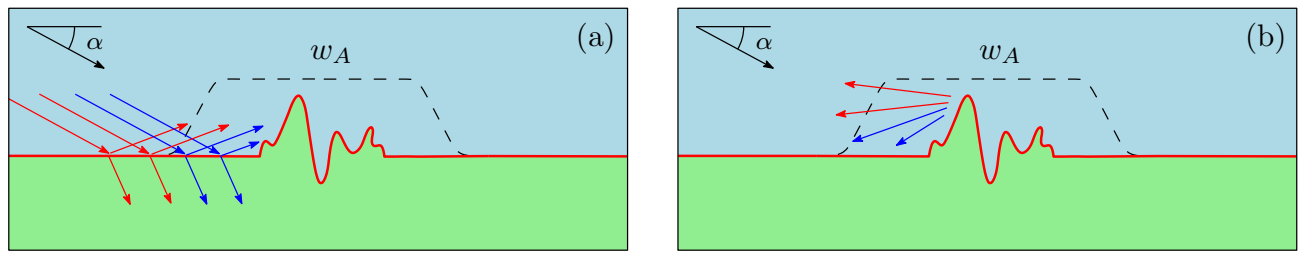

FIG. 3.3. Physical concepts underlying the WGF method.

3.4. Uniform superalgebraically fast convergence for all incidence angles. To address the difficulties demonstrated in Figure 3.2 we consider again the exact integral equation (3.3), and we replace the unknown density $\phi$ on the righthand side of this equation by the corresponding (known) density $\phi_{\Pi}^{f}$ associated with the problems of scattering and transmission of a plane-wave by a perfectly flat infinite interface $\Pi=\left\{\left(x_{1}, x_{2}\right) \in \mathbb{R}^{2}: x_{2}=0\right\}$. A closed form expression for the density $\phi_{\Pi}^{f}=\phi_{\Pi}^{f}\left(x_{1}\right)$ is derived in Appendix A of the supplementary materials, which are linked from the main article webpage. We thus obtain the approximate equation

$$
E \phi^{w}+T\left[W_{A} \phi^{w}\right]=\phi^{\mathrm{inc}}-T\left[\left(I-W_{A}\right) \phi_{\Pi}^{f}\right] \quad \text { on } \quad \Gamma_{A},
$$

whose solution $\phi^{w}$ is (see Remark 3.3) a superalgebraically close approximation of the exact solution $\phi$ which is valid throughout the region $\Gamma_{A} \cap\left\{w_{A}=1\right\}$ and which does not deteriorate as the incidence angle $\alpha$ tends to zero. 
In order to evaluate the term $T\left[\left(I-W_{A}\right) \phi_{\Pi}^{f}\right]$, we first consider the flat interface $\Pi$ and, in view of (2.7), we switch the integrations over $\Gamma$ of integrands involving $\left(I-W_{A}\right) \phi_{\Pi}^{f}$ into integrations over $\Pi$. To do this we rely on the fact that, since both $\phi_{\Pi}^{f}$ and $W_{A}$ are functions of $x_{1}$ only, these quantities and their product $\left(I-W_{A}\right) \phi_{\Pi}^{f}$ can be trivially extended to corresponding functions defined for all values of $\left(x_{1}, x_{2}\right) \in \mathbb{R}^{2}$ as constant functions of $x_{2}$ for each fixed $x_{1}$-which, in fact, vanish whenever $w_{A}=1$. The modification is thus straightforward: since $\left(I-W_{A}\right) \phi_{\Pi}^{f}$ vanishes on $\Gamma \backslash \Pi$ (at least for $A$ large enough), we may substitute the integration of an integrand equal to zero over the region $\Gamma_{A} \cap\left\{w_{A}=1\right\}$ by the integral over an integrand equal to zero over the region $\Pi \cap\left\{w_{A}=1\right\}$. We thus obtain

$$
T\left[\left(I-W_{A}\right) \phi_{\Pi}^{f}\right](\boldsymbol{x})=\widetilde{T}_{\Pi}\left[\left(I-W_{A}\right) \phi_{\Pi}^{f}\right](\boldsymbol{x}), \quad \boldsymbol{x} \in \Gamma,
$$

where, letting the layer potentials $\mathscr{S}_{j}^{\Pi}$ and $\mathscr{D}_{j}^{\Pi}(j=1,2)$ be given by

$$
\mathscr{S}_{j}^{\Pi}[\eta](\boldsymbol{x})=\int_{\Pi} G_{j}(\boldsymbol{x}, \boldsymbol{y}) \eta(\boldsymbol{y}) \mathrm{d} s_{\boldsymbol{y}} \quad \text { and } \quad \mathscr{D}_{j}^{\Pi}[\eta](\boldsymbol{x})=\int_{\Pi} \frac{\partial G_{j}}{\partial n_{\boldsymbol{y}}}(\boldsymbol{x}, \boldsymbol{y}) \eta(\boldsymbol{y}) \mathrm{d} s_{\boldsymbol{y}}
$$

for all $\boldsymbol{x} \in \mathbb{R}^{2}$, and defining the boundary integral operators

$$
\begin{aligned}
& \widetilde{S}_{j}^{\Pi}[\eta](\boldsymbol{x})=\mathscr{S}_{j}^{\Pi}[\eta](\boldsymbol{x}), \quad \widetilde{D}_{j}^{\Pi}[\eta](\boldsymbol{x})=\int_{\Pi} \frac{\partial G_{j}}{\partial n_{\boldsymbol{y}}}(\boldsymbol{x}, \boldsymbol{y}) \eta(\boldsymbol{y}) \mathrm{d} s_{\boldsymbol{y}}, \\
& \widetilde{N}_{j}^{\Pi}[\eta](\boldsymbol{x})=\frac{\partial \mathscr{D}_{j}^{\Pi}[\eta]}{\partial n}(\boldsymbol{x}), \quad \widetilde{K}_{j}^{\Pi}[\eta](\boldsymbol{x})=\int_{\Pi} \frac{\partial G_{j}}{\partial n_{\boldsymbol{x}}}(\boldsymbol{x}, \boldsymbol{y}) \eta(\boldsymbol{y}) \mathrm{d} s_{\boldsymbol{y}}, \\
& \boldsymbol{x} \in \Gamma, j=1,2,
\end{aligned}
$$

the operator $\widetilde{T}_{\Pi}$ is defined by

$$
\widetilde{T}_{\Pi}=\left[\begin{array}{cc}
\widetilde{D}_{2}^{\Pi}-\widetilde{D}_{1}^{\Pi} & -\widetilde{S}_{2}^{\Pi}+\nu \widetilde{S}_{1}^{\Pi} \\
\widetilde{N}_{2}^{\Pi}-\widetilde{N}_{1}^{\Pi} & -\widetilde{K}_{2}^{\Pi}+\nu \widetilde{K}_{1}^{\Pi}
\end{array}\right] .
$$

An important subtlety to be noted concerns the fact that $\widetilde{T}_{\Pi}$ maps density functions defined on $\Pi$ to functions defined on $\Gamma$.

Thus (3.4) becomes

$$
E \phi^{w}+T\left[W_{A} \phi^{w}\right]=\phi^{\text {inc }}-\widetilde{T}_{\Pi}\left[\phi_{\Pi}^{f}\right]+\widetilde{T}_{\Pi}\left[W_{A} \phi_{\Pi}^{f}\right] \quad \text { on } \quad \Gamma_{A} .
$$

Clearly the expression $\widetilde{T}_{\Pi}\left[W_{A} \phi_{\Pi}^{f}\right]$ can be evaluated by means of numerical integration over the bounded region $\Pi_{A}=\Pi \cap\left\{\left(x_{1}, x_{2}\right) \in \mathbb{R}^{2}: w_{A}\left(x_{1}\right) \neq 0\right\}$. As shown in Appendix A of the supplementary materials, on the other hand, the expression $\widetilde{T}_{\Pi}\left[\phi_{\Pi}^{f}\right]$ can be computed in closed form: 


$$
\widetilde{T}_{\Pi}\left[\phi_{\Pi}^{f}\right]=\phi^{\mathrm{inc}}-\left\{\begin{array}{cl}
{\left[u_{2}^{f}, \frac{(1+\nu)}{2} \frac{\partial u_{2}^{f}}{\partial n}\right]^{T}} & \text { on } \quad \Gamma \cap \Pi, \\
{\left[u^{f}, \frac{\partial u^{f}}{\partial n}\right]^{T}} & \text { on } \quad \Gamma \backslash \Pi .
\end{array}\right.
$$

The expressions on the right-hand side of (3.7) can thus be evaluated numerically throughout the surface $\Gamma_{A}$, and the corresponding bounded-domain integral equation can be solved by means of any available integral equation methodology - such as, for example, the highly accurate Nyström method $[11,18]$ we use.

To conclude this section, we demonstrate the fast and angle-independent convergence of $\phi^{w}$ to $\phi$ in Figure 3.4: clearly the value of $A$ required to obtain an accurate approximation of the exact solution has been reduced substantially and the errors are uniformly small as the incidence angle decreases to zero.

Remark 3.3. As mentioned above in this section, the solution of (3.4) is a uniformin- $\alpha$, superalgebraically close approximation of the exact solution $\phi$ throughout the curve $\Gamma_{A} \cap\left\{w_{A}=1\right\}$. This is established by means of a formal error analysis in section 4 . But a brief rationale may be provided within the geometrical-optics framework considered in the present section. Indeed, notice at first that, in view of the theory of asymptotic evaluation of integrals [4], the value of the surface potentials $\mathscr{S}_{j}$ and $\mathscr{D}_{j}$ in (2.5) which, in view of (2.3), are needed to evaluate the field at a point $\boldsymbol{x}$, can be obtained with superalgebraic accuracy by means of windowed integration in a region which contains all points of stationary phase [4, section 3.3]. But the points of stationary phase that arise for a given observation point $\boldsymbol{x}$ are precisely the points on the scattering surface where the rays reflect prior to their incidence upon $\boldsymbol{x}$. Thus, the windowed region in Figure 3.3, for example, contains (resp., does not contain) the points of stationary phase associated with the blue rays (resp., the red rays). But the contributions from red rays are reincorporated per (3.7), and, thus, all of the incidences that impact upon the curve $\Gamma_{A} \cap\left\{w_{A}=1\right\}$ on the first multiple-scattering iteration are taken into account with superalgebraically small errors. There remain fields that are not accounted for in (3.7), such as the field reflected by the windowed region which impacts outside of the windowed region. But these fields do not result in significant errors within the windowed region in any of the subsequent multiplescattering iterations: examination of the associated reflection points shows that only a superalgebraically small portion of the field reflected by the windowed region into the plane outside the windowed region reflects back into the windowed domain. We may thus conclude that the error arising from the substitution of $\phi$ by $\phi_{\Pi}^{f}$ ought to give rise to superalgebraically small errors in (3.7) throughout the curve $\Gamma_{A} \cap\left\{w_{A}=1\right\}$.

As mentioned in Remark 3.3, certain fields reflected by the windowed region, which do not affect the accuracy of the solution within the region $\Gamma_{A} \cap\left\{w_{A}=1\right\}$, are not taken into account within the formalism described in this section. These neglected fields do affect near fields and far fields in certain areas, however, as suggested by the ray description used throughout section 3. But, as shown in sections 3.5 and 3.6 below, the solution $\phi^{w}$ can be used to produce both the associated near field $u$ everywhere in space as well as far fields in all directions.

3.5. Field evaluation: Near fields. The discussion in Remark 3.3 extends directly to evaluation of near fields. Indeed, that remark tells us that substitution of $\phi$ by $w_{A} \phi^{w}+\left(1-w_{A}\right) \phi_{\Pi}^{f}\left(\phi_{\Pi}^{f}=\left[\varphi^{f}, \psi^{f}\right]^{T}\right)$ in the integral equation (3.3) leads to superalgebraically small errors $\boldsymbol{e}=\boldsymbol{\phi}-\boldsymbol{\phi}^{w}$ within the curve $\Gamma_{A} \cap\left\{w_{A}=1\right\}$. 

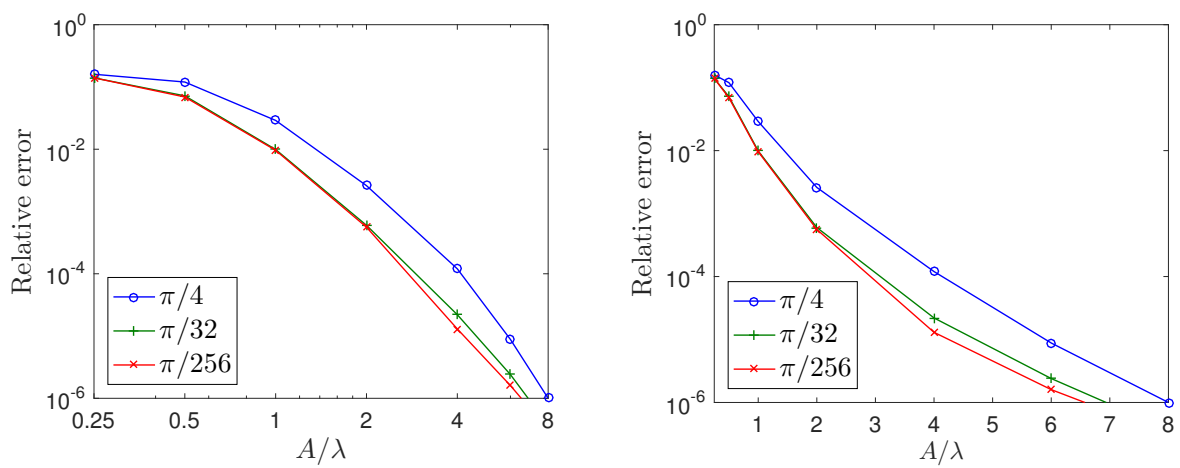

FIG. 3.4. Relative errors (see Remark 5.1) in the integral densities $\phi^{w}$ on the surface of the defect resulting from numerical solution of (3.7) for a semicircular bump-shaped defect, and for various window sizes and angles of incidence $\alpha=-\pi / 4$ (blue), $-\pi / 32$ (green), and $-\pi / 256$ (red) -including extremely shallow incidences. Left: log-log scale. Right: semi-log scale. Clearly, this version of the WGF method computes integral densities with superalgebraically high (but not exponential) accuracy uniformly for all angles of incidence (cf. Figure 3.2). Color is available online only.

Similar arguments can be used to establish that an analogous set of substitutions into the representation formula (2.3) produces the near field $u$ with superalgebraically small errors throughout the strip $[-c A, c A] \times \mathbb{R}$ (but see Remark 4.1). The necessary substitutions are as follows: substitution of $\left.u_{1}\right|_{\Gamma}$ and $\partial u_{1} /\left.\partial n\right|_{\Gamma}$ by $w_{A} \varphi^{w}+(1-$ $\left.w_{A}\right) \varphi^{f}-\left.u^{\mathrm{inc}}\right|_{\Gamma}$ and $\nu\left(w_{A} \psi^{w}+\left(1-w_{A}\right) \psi^{f}\right)-\partial u^{\mathrm{inc}} /\left.\partial n\right|_{\Gamma}$, respectively, in (2.3a), and substitution of $\left.u_{2}\right|_{\Gamma}$ and $\partial u_{2} /\left.\partial n\right|_{\Gamma}$ by $w_{A} \varphi^{w}+\left(1-w_{A}\right) \varphi^{f}$ and $w_{A} \psi^{w}+\left(1-w_{A}\right) \psi^{f}$, respectively, in (2.3b) (see (A.13) in Appendix A of the supplementary materials).

These substitutions together with the relation

$$
0=\mathscr{D}_{1}\left[\left.u^{\mathrm{inc}}\right|_{\Gamma}\right]-\mathscr{S}_{1}\left[\left.\frac{\partial u^{\mathrm{inc}}}{\partial n}\right|_{\Gamma}\right] \quad \text { in } \Omega_{1}
$$

(see [15]) leads to the expression

$$
u^{w}=\left\{\begin{array}{ccc}
u^{\text {inc }}+\mathscr{D}_{1}\left[w_{A} \varphi^{w}+\left(1-w_{A}\right) \varphi^{f}\right]-\nu \mathscr{S}_{1}\left[w_{A} \psi^{w}+\left(1-w_{A}\right) \psi^{f}\right] & \text { in } & \Omega_{1}, \\
u^{\|}-\mathscr{D}_{2}\left[w_{A} \varphi^{w}+\left(1-w_{A}\right) \varphi^{f}\right]+\mathscr{S}_{2}\left[w_{A} \psi^{w}+\left(1-w_{A}\right) \psi^{f}\right] & \text { in } & \Omega_{2}
\end{array}\right.
$$

for the approximate total near field $u^{w}$ in terms of the layer potentials defined in (2.5). After some manipulations similar to those presented in the derivation of (3.8), and using the relations (A.1), (A.2a), and (A.2b) of Appendix A in the supplementary materials, the formula in (3.9) is re-expressed in the forms

$$
\begin{aligned}
& u^{w}=\mathscr{D}_{1}\left[w_{A} \varphi^{w}\right]-\nu \mathscr{S}_{1}\left[w_{A} \psi^{w}\right] \\
& +\left\{\begin{array}{ccc}
u^{f}-\mathscr{D}_{1}^{\Pi}\left[w_{A} \varphi^{f}\right]+\nu \mathscr{S}_{1}^{\Pi}\left[w_{A} \psi^{f}\right] & \text { in } & \left\{x_{2}>0\right\}, \\
\left(1-\frac{w_{A}}{2}\right) u^{f}-D_{1}^{\Pi}\left[w_{A} \varphi^{f}\right]+\nu S_{1}^{\Pi}\left[w_{A} \psi^{f}\right] & \text { on } & \left\{x_{2}=0^{+}\right\}, \\
\frac{w_{A}}{2} u^{f}-D_{1}^{\Pi}\left[w_{A} \varphi^{f}\right]+\nu S_{1}^{\Pi}\left[w_{A} \psi^{f}\right] & \text { on } & \left\{x_{2}=0^{-}\right\}, \\
-\mathscr{D}_{1}^{\Pi}\left[w_{A} \varphi^{f}\right]+\nu \mathscr{S}_{1}^{\Pi}\left[w_{A} \psi^{f}\right] & \text { in } & \left\{x_{2}<0\right\},
\end{array}\right.
\end{aligned}
$$

Copyright $@$ ㅇ by SIAM. Unauthorized reproduction of this article is prohibited. 
within $\Omega_{1}$, and

(3.10b)

$$
\begin{aligned}
& u^{w}=-\mathscr{D}_{2}\left[w_{A} \varphi^{w}\right]+\mathscr{S}_{2}\left[w_{A} \psi^{w}\right] \\
& +\left\{\begin{array}{ccc}
\mathscr{D}_{2}^{\Pi}\left[w_{A} \varphi^{f}\right]-\mathscr{S}_{2}^{\Pi}\left[w_{A} \psi^{f}\right] & \text { in } & \left\{x_{2}>0\right\}, \\
\frac{w_{A}}{2} u^{f}+D_{2}^{\Pi}\left[w_{A} \varphi^{f}\right]-S_{2}^{\Pi}\left[w_{A} \psi^{f}\right] & \text { on } & \left\{x_{2}=0^{+}\right\}, \\
\left(1-\frac{w_{A}}{2}\right) u^{f}+D_{2}^{\Pi}\left[w_{A} \varphi^{f}\right]-S_{2}^{\Pi}\left[w_{A} \psi^{f}\right] & \text { on } & \left\{x_{2}=0^{-}\right\}, \\
u^{f}+\mathscr{D}_{2}^{\Pi}\left[w_{A} \varphi^{f}\right]-\mathscr{S}_{2}^{\Pi}\left[w_{A} \psi^{f}\right] & \text { in } & \left\{x_{2}<0\right\},
\end{array}\right.
\end{aligned}
$$

within $\Omega_{2}$, in terms of various surface potentials and operators defined either on $\Gamma$ or on $\Pi$-namely, the potentials $\mathscr{S}_{j}$ and $\mathscr{D}_{j}$ defined in (2.5), the potentials $\mathscr{S}_{j}^{\Pi}$ and $\mathscr{D}_{j}^{\Pi}$ defined in (3.5), and the operators $S_{j}^{\Pi}$ and $D_{j}^{\Pi}, j=1,2$, defined in (A.3) of Appendix A in the supplementary materials. Note that, by construction, the straight finite-length segments $\Pi \cap \Omega_{j}, j=1,2$, are contained in the region $\left\{-c A \leq x_{1} \leq c A\right\}$ for $A$ large enough; see Figures 2.1 and 3.6. Thus, for such values of $A$ the second and third expressions in both (3.10a) and (3.10b) give rise to an overall continuous and, indeed, smooth solution $u^{w}$, across the finite segments $\Pi \cap \Omega_{1}$ and $\Pi \cap \Omega_{2}$, respectively (Figure 2.1) - as it behooves a solution of the Helmholtz equation away from the dielectric interface $\Gamma$.

Figure 3.5 displays the total near field produced by means of both the WGF method and the layer-Green-function (LGF) method [26] for the solution of the problem of scattering of a plane-wave by a semicircular bump of radius $a=1$ in TEpolarization-with wavenumbers $k_{1}=10$ and $k_{2}=15$, and under two different incidence angles: $\alpha=-\pi / 2$ and $\alpha=-\pi / 6$. The WGF solutions were obtained from the integral equation (3.7) followed by evaluation of field values on the basis of (3.10) (but see also the last paragraph is section 3.6 in regards to near-field evaluation with higher accuracy and/or overextended regions). The absolute errors (see Remark 5.1) in the WGF solutions displayed in Figures 3.5a and 3.5c over the complete range shown are $1 \cdot 10^{-4}$ and $2 \cdot 10^{-4}$, respectively.

3.6. Field evaluation: Far fields. In view of the analysis in section 4 it follows that formulae (3.10) do not generally provide an accurate approximation of either far fields or near fields outside bounded subsets of $[-c A, c A] \times \mathbb{R}$ (see, in particular, Remark 4.1). In order to tackle this difficulty we consider the boundary $S$ of a disc $D$ such as the one depicted in Figure 3.6. The curve $S$ encloses the portion of $\Gamma$ that differs from the flat interface $\Pi$; as indicated above, superalgebraic convergence of the fields $u_{1}$ and $u_{2}$ takes place everywhere on and within such a curve $S$. Then, application of the Green identities, integration over the region exterior to $S$, and use of the layer Green function (see (B.6) in Appendix B of the supplementary materials) leads to the integral representation

$$
u^{s}(\boldsymbol{x})=\int_{S}\left\{\frac{\partial G}{\partial n_{\boldsymbol{y}}}(\boldsymbol{x}, \boldsymbol{y}) u^{s}(\boldsymbol{y})-G(\boldsymbol{x}, \boldsymbol{y}) \frac{\partial u^{s}}{\partial n}(\boldsymbol{y})\right\} \mathrm{d} s_{\boldsymbol{y}}
$$

of scattered field $u^{s}=u-u^{f}$, which is valid for $\boldsymbol{x}$ everywhere outside $S$. Here $G$ denotes the layer Green function for the Helmholtz equation with wavenumbers $k_{1}$ in $\left\{x_{2} \geq 0\right\}$ and $k_{2}$ in $\left\{x_{2}<0\right\}$ (see Appendix B of the supplementary materials). Note that the necessary values of the scattered field $u^{s}$ and its normal derivative on $S$ can 


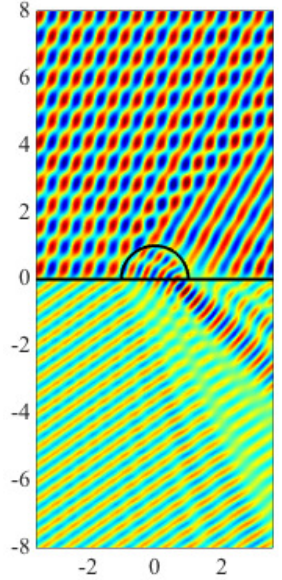

(a) WGF method

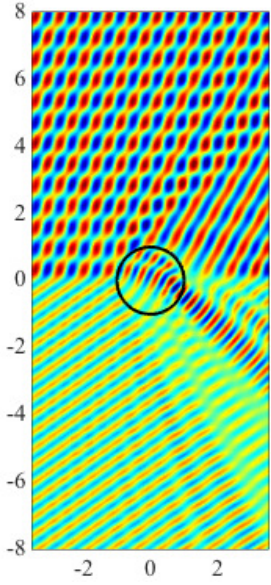

(b) LGF method

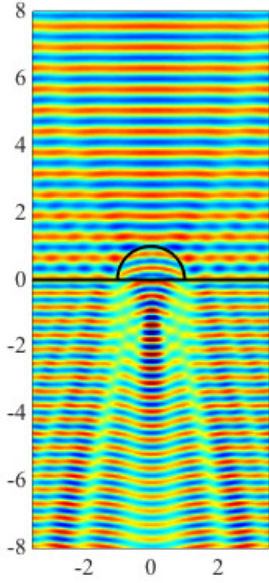

(c) WGF method

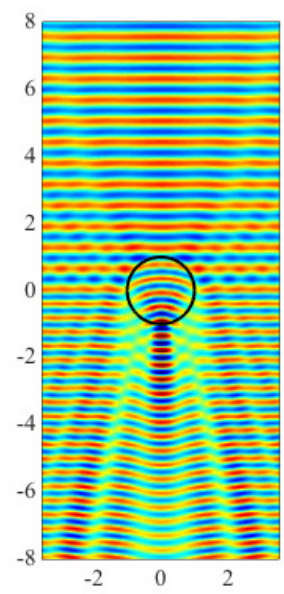

(d) LGF method

FIG. 3.5. Real parts of the total fields produced by the WGF method and the LGF method for the problem of scattering of a plane-wave by a semicircular bump. Figures 3.5a and 3.5b: $\alpha=-\pi / 6$. Figures 3.5c and 3.5d: $\alpha=-\pi / 2$. The width of the support of the selected window function is $2 A=16 \lambda \approx 10.053$ in all these calculations. The black lines represent the domains where the respective integral equation formulations are posed. (Note that in addition to the surface of the bump itself, the LGF method [26] entails discretization of a certain transparent boundary in the lower half-plane-so that, in the present bump cases, for example, the LGF integral equations are actually posed on the full circles depicted in Figures (b) and (d)).

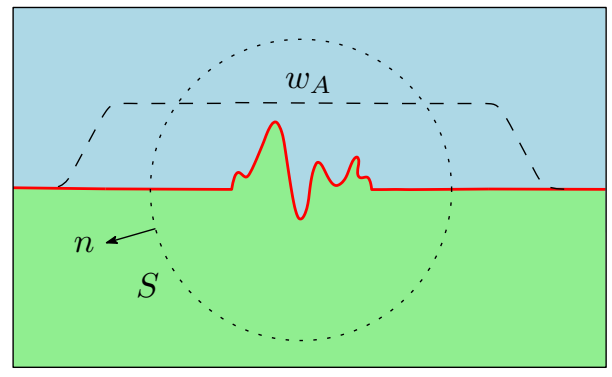

FIG. 3.6. Curve $S$ utilized in (3.11).

be computed directly utilizing (3.10), since, by construction, $S$ lies inside the region where (3.10) provides an accurate approximation of the total field $u$.

The far-field pattern $u_{\infty}(\hat{\boldsymbol{x}})$, which is related to the scattered field by the asymptotic formula

$$
u^{s}(\boldsymbol{x})=\frac{\mathrm{e}^{i k_{1}|\boldsymbol{x}|}}{\sqrt{|\boldsymbol{x}|}} u_{\infty}(\hat{\boldsymbol{x}})+\mathcal{O}\left(|\boldsymbol{x}|^{-3 / 2}\right), \quad|\boldsymbol{x}| \rightarrow \infty, \quad \hat{\boldsymbol{x}}=\frac{\boldsymbol{x}}{|\boldsymbol{x}|},
$$

can be produced by replacing $G$ and its normal derivative in (3.11) by their corresponding leading-order asymptotic expansions as $|\boldsymbol{x}| \rightarrow \infty$. As shown in Appendix B of the supplementary materials, the first-order term of the asymptotic expansion of the layer Green function in a given direction $\hat{\boldsymbol{x}}=(\cos \theta, \sin \theta), 0<\theta<\pi$, can be obtained by the method of steepest descent; see [3]. Substitution of the result in 
(3.11) yields the expression

$$
u_{\infty}(\hat{\boldsymbol{x}})=\int_{S}\left\{n(\boldsymbol{y}) \cdot \boldsymbol{H}_{\infty}(\hat{\boldsymbol{x}}, \boldsymbol{y}) u^{s}(\boldsymbol{y})-G_{\infty}(\hat{\boldsymbol{x}}, \boldsymbol{y}) \frac{\partial u^{s}}{\partial n}(\boldsymbol{y})\right\} \mathrm{d} s_{\boldsymbol{y}}
$$

for the far field $u_{\infty}(\hat{\boldsymbol{x}})$, where the far-field kernels $G_{\infty}$ and $\boldsymbol{H}_{\infty}$ are given in (B.13) and (B.14) of Appendix B in the supplementary materials, respectively. Thus, unlike the layer Green function $G$ itself for small values of $|\boldsymbol{x}-\boldsymbol{y}|$, the far-field associated with $G$ can be computed inexpensively by means of the explicit expressions (B.13) and (B.14). Figure 3.7 provides a comparison of the far-field patterns computed using the LGF and WGF methods for the problem considered in section 3.5.
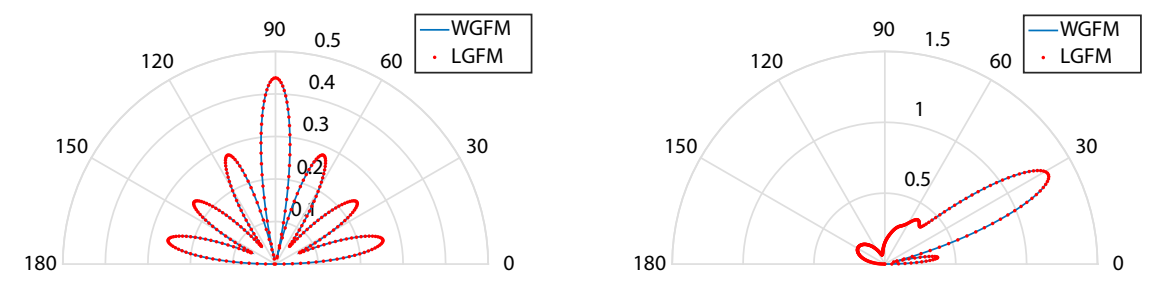

FIG. 3.7. Far-field patterns obtained using the LGF method [26] (red dotted curve) and the WGF method (continuous blue line) for the solution of the problem of scattering considered in this section at incidences $\alpha=-\pi / 2$ (left) and $\alpha=-\pi / 6$ (right). Color is available online only.

In view of section 3.5 and the discussion above in this section, (3.10) and (3.12) can be used to accurately and efficiently evaluate near fields and far fields, respectively. These are typically the quantities of interest in scattering simulations involving layered media. The evaluation of the fields in an intermediate region, such as the complement of a bounded domain within the strip $[-c A, c A]$ (where (3.10) yields an accurate approximation), can be approximated efficiently on the basis of (3.11). Indeed, in such cases, for which source points $\boldsymbol{y}$ lie on $S$ and observation points $\boldsymbol{x}$ are at a large distance away from $S$, the Sommerfeld integrals in (B.6) of Appendix B in the supplementary materials (which by Cauchy's theorem can be expressed in terms of complex contour integrals with highly oscillatory and/or exponentially decaying integrands) can be obtained rapidly by means of asymptotic numerical methods $[2$, 6 ] - based on localization around critical points and the method of steepest descents in very small regions around saddle points.

4. Formal error analysis. A formal multiple-scattering error analysis introduced in section 4.4 validates the ray-based discussions presented in section 3 . The arguments presented in section 4.4 rely on the WGF approximation properties for certain simple "obstacle-free problems": the problem of scattering by a planar interface in the absence of a defect or obstacle, and including point-source incident fields. Useful insights into these regards can be obtained by consideration of obstacle-free problems under plane-wave incident fields with possibly complex wavevectors - which, via integration, can be used to represent an arbitrary point source by complex contour evaluation of the integral in (4.12). The preliminary discussion concerning plane-wave incidence is advantageous in a number of ways, as (1) the error of the complete range of relevant plane-wave approximations is dominated by the "worst-case" errors which arise for real wavevectors at normal incidence (see, e.g., Figures 4.1 and 4.2); (2) the worst-case errors can be characterized by a single parameter (namely the number of 
wavelengths $A / \lambda=k_{1} A /(2 \pi)$ contained in the windowed region); and, as shown elsewhere, (3) the windowed obstacle-free problem lends itself more directly to analysis under plane-wave incidence - since, unlike the WGF solutions for point-source problems, the plane-wave WGF solutions (for either a real or complex incident wavevector) can be expressed as the product of the incident field times a function whose derivatives decay as $A \rightarrow \infty$.

Section 4.1 presents a discussion of the WGF method for the plane-wave obstaclefree problem, and sections 4.2 and 4.3 further illustrate and augment these discussions through a variety of numerical examples for both plane-wave and point-source illumination. As mentioned above, our formal multiple-scattering error analysis is then presented in section 4.4.

\subsection{WGF solution of plane-wave-illuminated planar interface.}

4.1.1. WGF error sources. With reference to (4.12) and its complex contour representation used in section 4.3 , for a given point $\boldsymbol{y}=\left(y_{1}, y_{2}\right), y_{2}>0$, and complex wavevector $\left(\xi,-i \gamma_{1}(\xi)\right)$ we consider the problem of scattering by a dielectric half-plane illuminated by a (generalized) plane wave $u_{\xi}^{\text {inc }}$ of the form

$$
u_{\xi}^{\mathrm{inc}}(\boldsymbol{x})=\frac{\mathrm{e}^{i \xi\left(x_{1}-y_{1}\right)-\gamma_{1}(\xi)\left|x_{2}-y_{2}\right|}}{\gamma_{1}(\xi)}=C_{\xi} \mathrm{e}^{i \xi x_{1}+\gamma_{1}(\xi) x_{2}}
$$

$\left(C_{\xi}=\frac{\mathrm{e}^{-i \xi y_{1}-\gamma_{1}(\xi) y_{2}}}{\gamma_{1}(\xi)}\right)$ with $y_{2}>x_{2}\left(x_{2}=0\right.$ for the planar interface considered in this section). Here $\gamma_{1}(\xi)=\sqrt{\xi^{2}-k_{1}^{2}}=\sqrt{\xi-k_{1}} \sqrt{\xi+k_{1}}$ with branches selected as indicated in Appendix B of the supplementary materials. Note that the quantity $\left|x_{2}-y_{2}\right|$ in (4.1) equals $y_{2}-x_{2}$ under the $y_{2}>x_{2}$ assumption included in the equation, of course, but the absolute values are kept in order to match the form of the integrand in the plane-wave integral expression (4.12) for a point-source incident field.

Following section 2 we obtain the integral equation formulation

$$
E \phi_{\xi}+T_{\Pi}\left[\phi_{\xi}\right]=\phi_{\xi}^{\text {inc }} \quad \text { on } \Pi
$$

for the present problem, where the operator $T_{\Pi}$ is defined as in (2.7)-(2.8) with $\Gamma=\Pi$, and where letting $\phi_{\xi}=\left(\varphi_{\xi}, \psi_{\xi}\right)$, the right-hand-side is given by

$$
\phi_{\xi}^{\text {inc }}=\left[\left.u_{\xi}^{\text {inc }}\right|_{\Pi},\left.\frac{\mathrm{d} u_{\xi}^{\text {inc }}}{\mathrm{d} x_{2}}\right|_{\Pi}\right]^{T} .
$$

The solution of (4.2) can be obtained by letting $\xi=k_{1} \cos \alpha$ in the relevant expressions in Appendix A of the supplementary materials: with this identification we have $\boldsymbol{\phi}_{\Pi}^{\text {inc }}=$ $\phi_{\xi}^{\text {inc }} / C_{\xi}$, and, thus, the exact solution of (4.2) coincides with (A.13) in Appendix A of the supplementary materials. In terms of $\xi$ we thus have

$$
\varphi_{\xi}(t)=C_{\xi} T_{\xi} \mathrm{e}^{i \xi t} \quad \text { and } \quad \psi_{\xi}(t)=\frac{\gamma_{1}(\xi) C_{\xi}\left(2-T_{\xi}\right)}{\nu} \mathrm{e}^{i \xi t},
$$

where $T_{\xi}=\frac{2 \gamma_{1}(\xi)}{\gamma_{1}(\xi)+\nu \gamma_{2}(\xi)}$ and where $\gamma_{2}(\xi)=\sqrt{\xi^{2}-k_{2}^{2}}$ (with a choice of branches as specified in Appendix B of the supplementary materials).

Our analysis relies on the use of a certain approximate solution $\boldsymbol{\phi}_{\xi}^{w}=\left[\varphi_{\xi}^{w}, \psi_{\xi}^{w}\right]^{T}$ of (4.2) which is obtained by means of the windowing approximation but without use of a correction term akin to $T\left[\left(I-W_{A}\right) \phi_{\Pi}^{f}\right]$ in (3.4): the density $\phi_{\xi}^{w}$ satisfies

$$
E \phi_{\xi}^{w}+T_{\Pi}\left[W_{A} \phi_{\xi}^{w}\right]=\phi_{\xi}^{\text {inc }} \quad \text { on } \quad \Pi_{A},
$$


where $\Pi_{A}=\Pi \cap\{-A \leq x \leq A\}$. As shown in what follows, both $\phi_{\xi}^{w}$ itself and the reflected and transmitted fields it produces according to

$$
u_{\xi}^{w}(\boldsymbol{x})=\left\{\begin{array}{cc}
\mathscr{D}_{1}^{\Pi}\left[w_{A} \varphi_{\xi}^{w}\right](\boldsymbol{x})-\nu \mathscr{S}_{1}^{\Pi}\left[w_{A} \psi_{\xi}^{w}\right](\boldsymbol{x}), & \left\{x_{2}>0\right\}, \\
-\mathscr{D}_{2}^{\Pi}\left[w_{A} \varphi_{\xi}^{w}\right](\boldsymbol{x})+\mathscr{S}_{2}^{\Pi}\left[w_{A} \psi_{\xi}^{w}\right](\boldsymbol{x}), & \left\{x_{2}<0\right\}
\end{array}\right.
$$

(cf. (2.3)), are highly accurate for a certain range of complex values of $\xi$ - a fact that is relevant in the analysis presented at various points in section 4.4.

In order to appreciate the need for consideration of complex values of $\xi$ we first study the errors that result from the use of the approximate equation (4.4) for a given real value of $\xi$. To gain an insight into the extent of such errors we subtract (4.4) from (4.2), and we thus find that the error $\boldsymbol{e}_{\xi}^{w}=\phi_{\xi}-\phi_{\xi}^{w}$ satisfies the equation

$$
E \boldsymbol{e}_{\xi}^{w}+T_{\Pi}\left[W_{A} \boldsymbol{e}_{\xi}^{w}\right]=T_{\Pi}\left[\left(I-W_{A}\right) \phi_{\xi}\right] \quad \text { on } \quad \Pi_{A} .
$$

The error source $T_{\Pi}\left[\left(I-W_{A}\right) \phi_{\xi}\right]$ provides an important indication of the expected error sizes. For definiteness we focus on one of the various contributions to this quantity, namely $T_{12}\left[\left(1-w_{A}\right) \psi_{\xi}\right]$ (see $\left.(2.7)\right)$; all other contributions can be treated similarly.

The error source term $T_{12}\left[\left(1-w_{A}\right) \psi_{\xi}\right]$ is given by a linear combination of the single-layer potentials

$$
\begin{aligned}
S_{j}\left[\left(1-w_{A}(\cdot)\right) \mathrm{e}^{i \xi \cdot}\right](t)= & \frac{i}{4} \int_{-\infty}^{\infty} H_{0}^{(1)}\left(k_{j}|t-\tau|\right)\left[1-w_{A}(\tau)\right] \mathrm{e}^{i \xi \tau} \mathrm{d} \tau \\
= & \frac{i}{4} \int_{-\infty}^{-c A} H_{0}^{(1)}\left(k_{j}|t-\tau|\right) \widetilde{w}_{A}(\tau) \mathrm{e}^{i \xi \tau} \mathrm{d} \tau \\
& +\frac{i}{4} \int_{c A}^{\infty} H_{0}^{(1)}\left(k_{j}|t-\tau| \widetilde{w}_{A}(\tau) \mathrm{e}^{i \xi \tau} \mathrm{d} \tau\right.
\end{aligned}
$$

$(j=1,2)$. We consider the last term first. Introducing the change of variables $t=A s$ and $\tau=A \sigma$ the last integral in (4.6) is expressed in the form

$$
A \mathrm{e}^{-i A k_{j} s} \int_{c}^{\infty} h_{0}\left(A k_{j}|s-\sigma|\right) \widetilde{w}_{1}(\sigma) \mathrm{e}^{i A \sigma\left(k_{j}+\xi\right)} \mathrm{d} \sigma,
$$

which we estimate in what follows for values of $s$ throughout the interval $[-c, c]$ (that is, throughout the region $\left\{s: w_{A}(A s)=1\right\}=\{s: w(s ; c, 1)=1\}$ ) under the assumption $k_{j}+\xi \neq 0$. Here, given $k>0$ and $d>0$, the (nonoscillatory) function $h_{\ell}(x)=\mathrm{e}^{-i x} H_{\ell}^{(1)}(x)(\ell \geq 0, x \geq 0)$ satisfies the estimates

$$
\left|\left(\frac{\mathrm{d}}{\mathrm{d} x}\right)^{m}\left[h_{\ell}(k x)\right]\right| \leq\left\{\begin{array}{cll}
C_{m, \ell}(k x)^{-1 / 2} x^{-m} & \text { if } \quad k x \geq d \\
C_{m, \ell}(k x)^{-\ell} x^{-m} & \text { if } \quad 0<k x<d \text { and } m+\ell>0 \\
C_{0,0}(1+|\log k x|) & \text { if } \quad 0<k x<d \text { and } m=\ell=0
\end{array}\right.
$$

for some constants $C_{m, \ell}>0, m \geq 0$. (This follows from the well-known asymptotic expression [19, section 5.11] for the Hankel function; see also [14, Lemma 1].)

To estimate the error source (4.7) for a given $\xi$ we note that, in view of the absence points of stationary phase in the region $\{|\sigma|>c\}$, after $m$ integrations by parts we obtain

$$
\frac{(-1)^{m} A \mathrm{e}^{-i A k_{j} s}}{\left[i A\left(k_{j}+\xi\right)\right]^{m}} \int_{c}^{\infty}\left(\frac{\mathrm{d}}{\mathrm{d} \sigma}\right)^{m}\left[h_{0}\left(A k_{j}|s-\sigma|\right) \widetilde{w}_{1}(\sigma)\right] \mathrm{e}^{i A \sigma\left(k_{j}+\xi\right)} \mathrm{d} \sigma
$$

Copyright $@$ by SIAM. Unauthorized reproduction of this article is prohibited. 
since all the boundary contributions vanish. This can be checked by taking into account that (a) the function $\widetilde{w}_{1}$ and its derivatives vanish at $\sigma=c$, and that, in view of (4.8), (b) the function $h_{0}\left(A k_{j}|s-\sigma|\right)$ and its derivatives decay as $\sigma \rightarrow \infty$.

The integration-by-parts procedure used above requires that for all $s \in[-c, c]$ the integrand in (4.7) be an infinitely smooth function of $\sigma$ throughout the integration domain. This is straightforward for $s \in[-c, c)$, and it holds for $s=c$ as well-in spite of the fact that, for $s=c, h_{0}\left(A k_{j}(\sigma-s)\right)$ and its derivatives are singular at $\sigma=c$-since the window function $\widetilde{w}_{1}(\sigma)=1-w(\sigma ; c, 1)$ vanishes along with all of its derivatives at the endpoint $\sigma=c$.

Utilizing (4.8) it additionally follows that the value of the integral in (4.9) remains bounded for all $A>0$. We therefore conclude from (4.9) that for $|s| \leq c$ the last integral in (4.6) is a superalgebraically small quantity (it decays faster than any integer power of $1 / A$ ) as long as $k_{j}+\xi \neq 0$. Similarly, it can be shown the next-to-last integral in (4.6) is superalgebraically small as long as $k_{j}-\xi \neq 0$, and thus we conclude that provided $k_{j} \pm \xi \neq 0$ the term $T_{\Pi}\left[\left(I-W_{A}\right) \phi_{\xi}\right]$ decreases superalgebraically fast as $A \rightarrow \infty$ within the interval $[-c A, c A]$.

Clearly, increasingly larger values of $A$ are necessary to keep the error-source term (4.9) below a given tolerance as $\left|k_{j} \pm \xi\right| \rightarrow 0$. The last column of Table 4.1 demonstrates that, as expected, the corresponding errors $\boldsymbol{e}_{\xi}^{w}$ arising in the integral equation (4.4) exhibit slow convergence for small values of $\left|k_{j} \pm \xi\right|$ as well. Fortunately, however, small values of $\left|k_{j} \pm \xi\right|$ can be completely avoided in the analysis presented in section 4.4 by representing point sources as a contour integral in the complex $\xi$ plane. A discussion concerning the errors $\boldsymbol{e}_{\xi}^{w}$ that arise in the integral equation (4.4) as a result of the aforementioned errors sources, but with allowance for complex values of $\xi$, is presented in the following section.

4.1.2. Error estimation for complex values of $\xi$. As mentioned in section 4.1.1, the formal multiple-scattering error analysis presented in section 4.4, which applies to the case in which the WGF method is used to produce the solutions of problems of scattering by a bounded obstacle in the presence of a planar dielectric layer, can be established provided corresponding estimates for the error $\boldsymbol{e}_{\xi}^{w}$ on $[-c A, c A]$ for the obstacle-free problem are available for certain complex values of $\xi$. Under certain smoothness assumptions on $\phi_{\xi}^{w}$, which have been verified numerically, such estimates on $\boldsymbol{e}_{\xi}^{w}$ can be obtained (for $\xi \in \mathbb{C}, \operatorname{Re} \xi \cdot \operatorname{Im} \xi \leq 0, \xi \neq \pm k_{j}$ ) on the basis of the "improper" integral equation

$$
E \boldsymbol{e}_{\xi}+T_{\Pi}\left[\boldsymbol{e}_{\xi}\right]=\left(I-W_{A}\right)\left\{\boldsymbol{\phi}_{\xi}^{\mathrm{inc}}-T_{\Pi}\left[W_{A} \boldsymbol{\phi}_{\xi}^{w}\right]\right\} \quad \text { on } \quad \Pi
$$

that is satisfied by a new error density $\boldsymbol{e}_{\xi}=\phi_{\xi}-W_{A} \phi_{\xi}^{w}$. Note that, by definition, $\boldsymbol{e}_{\xi}=\boldsymbol{e}_{\xi}^{w}$ on $[-c A, c A]$.

We call this integral equation improper in view of its infinite-domain exponentially growing integrand. Notice, for example, that the related integral equation (4.2), which for such complex values of $\xi$ entails a closely related exponentially growing integrand, admits the exact solution (4.3). Equation (4.2) represents the most singular term in (4.10); the remaining terms do not present difficulties. (The numerical values of $\boldsymbol{e}_{\xi}$ presented in Table 4.1 for complex values of $\xi$, for example, were evaluated as the difference of the numerical WGF solution and the exact solution (4.3).) The integral equation (4.10) could alternatively (and more generally) be interpreted via an appeal to analytic contour integration in the complex plane, following the work [16] and associated literature. 
Relying on (1) the convolution character of the operator $T$ to explicitly solve (4.10) by means of Fourier transform techniques, together with (2) an extension of the integration-by-parts arguments presented in section 4.1.1 to complex values of $\xi$, and (3) the aforementioned smoothness assumptions on $\phi_{\xi}^{w}$, it can be shown that the error $\boldsymbol{e}_{\xi}$ is superalgebraically small throughout the region $[-c A, c A]$. Additionally, the error estimates can be extended to the values of the scattered fields in certain regions around the windowed domain (but see also Remark 4.1). The rigorous proofs of these estimates lie outside of the scope of this paper and will be presented elsewhere. The next section presents a variety of numerical results demonstrating that, as suggested in the present section, the WGF method for the obstacle-free case does give rise to superalgebraically convergent integral-equation solutions and scattered fields.

Remark 4.1. It is important to note that the aforementioned near-field convergence is not uniform in the strip $[-c A, c A] \times \mathbb{R}$ : for points in this region with larger and larger values of $x_{2}$, correspondingly larger and larger values of $A>0$ are necessary to reach a prescribed error tolerance; see also Figures 4.1, 4.2, and 4.4.

Errors $\left\|\boldsymbol{e}_{\xi}\right\|_{L^{\infty}([-c A, c A])}=\left\|\boldsymbol{e}_{\xi}^{w}\right\|_{L^{\infty}([-c A, c A])}=\left\|\phi_{\xi}-\phi_{\xi}^{w}\right\|_{L^{\infty}([-c A, c A])}$ for various window sizes and values of the parameter $\xi$ obtained in the solution of the problem of scattering of $u_{\xi}^{\text {inc }}$, defined in (4.1) for $\boldsymbol{y}=(0,1)$, by a dielectric plane for wavenumbers $k_{1}=20$ and $k_{2}=40$. As demonstrated by the $\xi=19.99$ column in this table, which is included for completeness, slow convergence takes place for values of $\xi$ for which $\left|k_{j} \pm \xi\right|$ is small. As indicated in the text, however, such situations are bypassed in the error analysis presented in section 4.4 by an appropriate selection of complex integration contours.

\begin{tabular}{c|c|c|c|c|c}
\hline & \multicolumn{5}{|c}{ Error } \\
\hline$A$ & $\xi=0$ & $\xi=20-i$ & $\xi=40-i$ & $\xi=50$ & $\xi=19.99$ \\
\hline $2 \lambda$ & $1.26 \cdot 10^{-2}$ & $1.06 \cdot 10^{-3}$ & $2.10 \cdot 10^{-17}$ & $1.42 \cdot 10^{-22}$ & $\mathbf{2 . 0 4} \cdot \mathbf{1 0}-\mathbf{0}$ \\
\hline $2^{2} \lambda$ & $3.29 \cdot 10^{-3}$ & $5.72 \cdot 10^{-4}$ & $1.04 \cdot 10^{-17}$ & $9.21 \cdot 10^{-23}$ & $\mathbf{1 . 9 5} \cdot \mathbf{1 0}^{-\mathbf{0}}$ \\
\hline $2^{3} \lambda$ & $5.04 \cdot 10^{-4}$ & $8.98 \cdot 10^{-6}$ & $1.35 \cdot 10^{-19}$ & $1.38 \cdot 10^{-24}$ & $\mathbf{3 . 7 0} \cdot \mathbf{1 0}^{-\mathbf{1}}$ \\
\hline $2^{4} \lambda$ & $2.95 \cdot 10^{-5}$ & $7.91 \cdot 10^{-7}$ & $8.28 \cdot 10^{-21}$ & $1.31 \cdot 10^{-25}$ & $\mathbf{2 . 3 6} \cdot \mathbf{1 0}^{-\mathbf{1}}$ \\
\hline $2^{5} \lambda$ & $5.57 \cdot 10^{-7}$ & $1.57 \cdot 10^{-8}$ & $3.68 \cdot 10^{-21}$ & $8.65 \cdot 10^{-27}$ & $\mathbf{1 . 4 5} \cdot \mathbf{1 0}^{-\mathbf{1}}$ \\
\hline
\end{tabular}

4.2. Obstacle-free problem under plane-wave incidence: Numerical illustrations. To illustrate the WGF approximation properties considered in section 4.1, here we present Table 4.1 and Figures 4.1 and 4.2. With reference to the notations in that section, Table 4.1 displays the maximum throughout $[-c A, c A]$ of the numerical errors $\boldsymbol{e}_{\xi}^{w}$ that result for incident waves $u_{\xi}^{\mathrm{inc}}$ with various relevant complex values of $\xi$ (cf. (4.1) and the associated text). As demonstrated in these experiments, $\phi_{\xi}^{w}$ does indeed approximate $\phi_{\xi}$ with superalgebraically small errors within the region $[-c A, c A]$ for $\xi \in \mathbb{C}, \operatorname{Re} \xi \cdot \operatorname{Im} \xi \leq 0$, such that $\xi \neq \pm k_{1}$ or $\xi \neq \pm k_{2}$. In accordance with the discussion in section 4.1 , it is clear that large values of $A$ are generally required for convergence to a given error whenever $\left|k_{j} \pm \xi\right|$ is small. But this does not impact upon the multiple-scattering error analysis presented in section 4.4, since the complex integration contour used in that section (Figure 4.3) completely avoids a neighborhood of the points $\xi \neq \pm k_{1}$.

The errors introduced by the obstacle-free WGF method in the scattered field for values of $\xi$ along the aforementioned complex contour are also considered in the context of the multiple-scattering error analysis presented in section 4.4. The field errors resulting for a few relevant complex-wavevector incidences $\xi$ are presented in 


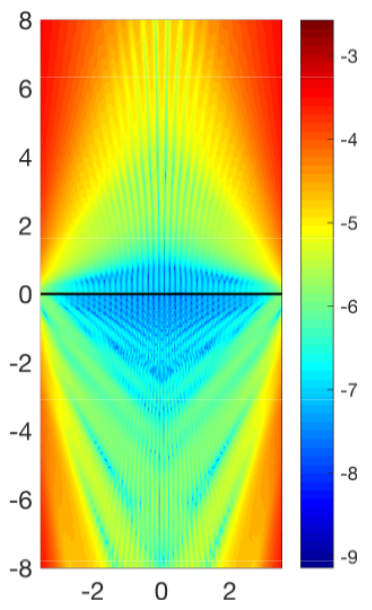

(a) $\xi=0$.

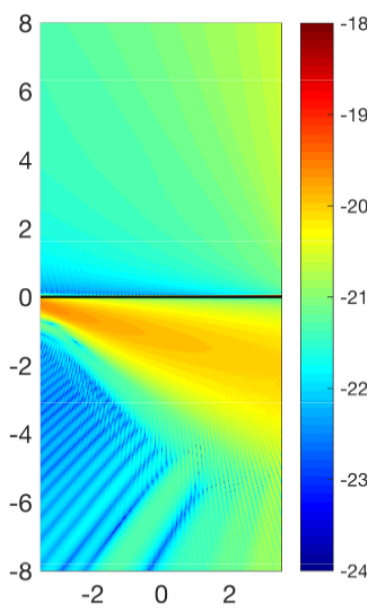

(c) $\xi=40-i$.

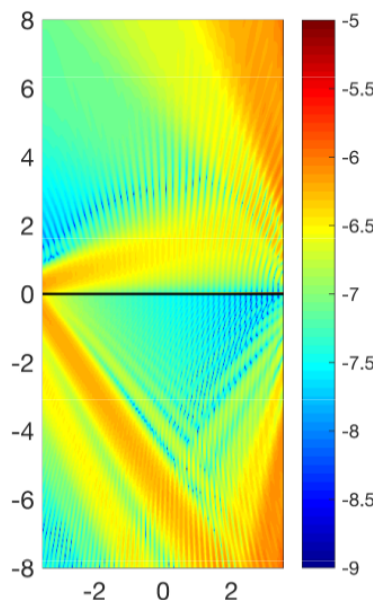

(b) $\xi=20-i$.

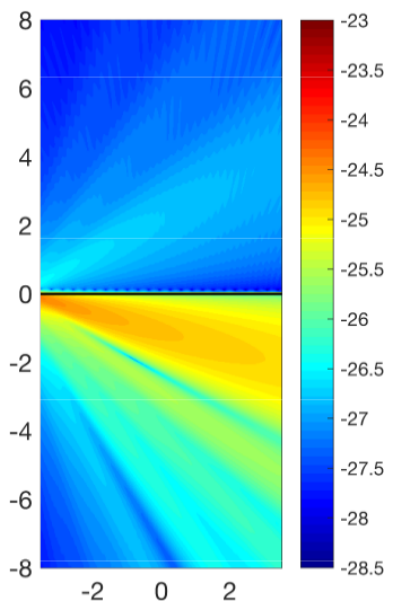

(d) $\xi=50$.

FIG. 4.1. Logarithm of the field errors $\log _{10}\left|u_{\xi}(\boldsymbol{x})-u_{\xi}^{w}(\boldsymbol{x})\right|$ obtained by means of the WGF method with $A=16 \lambda\left(\lambda=2 \Pi / k_{1}\right)$ for the problem of scattering of $u_{\xi}^{\text {inc }}$ defined in (4.1) for $\boldsymbol{y}=(0,1)$, by a dielectric plane for various values of $\xi$ and wavenumbers $k_{1}=20$ and $k_{2}=40$. The quantity $u_{\xi}^{w}$ is defined in (4.5), and $u_{\xi}$ equals the exact reflected field $C_{\xi}\left(T_{\xi}-1\right) \mathrm{e}^{i \xi x_{1}-\gamma_{1}(\xi) x_{2}}$ in the upper halfplane $\left\{x_{2}>0\right\}$ and the exact transmitted field $C_{\xi} T_{\xi} \mathrm{e}^{i \xi x_{1}+\gamma_{2}(\xi) x_{2}}$ in the lower half-plane $\left\{x_{2}<0\right\}$.

Figure 4.1. Note the extremely small field values that arise for the relatively small window size $A=16 \lambda$. In fact, the largest such errors take place for the case $\xi=0$ which corresponds to an incident field with a real wavevector (that is, a physically realizable incident field) under normal incidence. As demonstrated in Figure 4.2, even in this case fast convergence takes place. For example, the use of windows of sizes $A=8 \lambda, A=16 \lambda$, and $A=32 \lambda$ suffices to produce solutions with errors of the order of $10^{-3.68}, 10^{-5.18}$, and $10^{-7.04}$, respectively, on a certain representative segment in space (details are presented in the figure caption).

4.3. Obstacle-free problem under point-source incidence: Numerical illustrations. To conclude this section we study the errors introduced by the WGF 

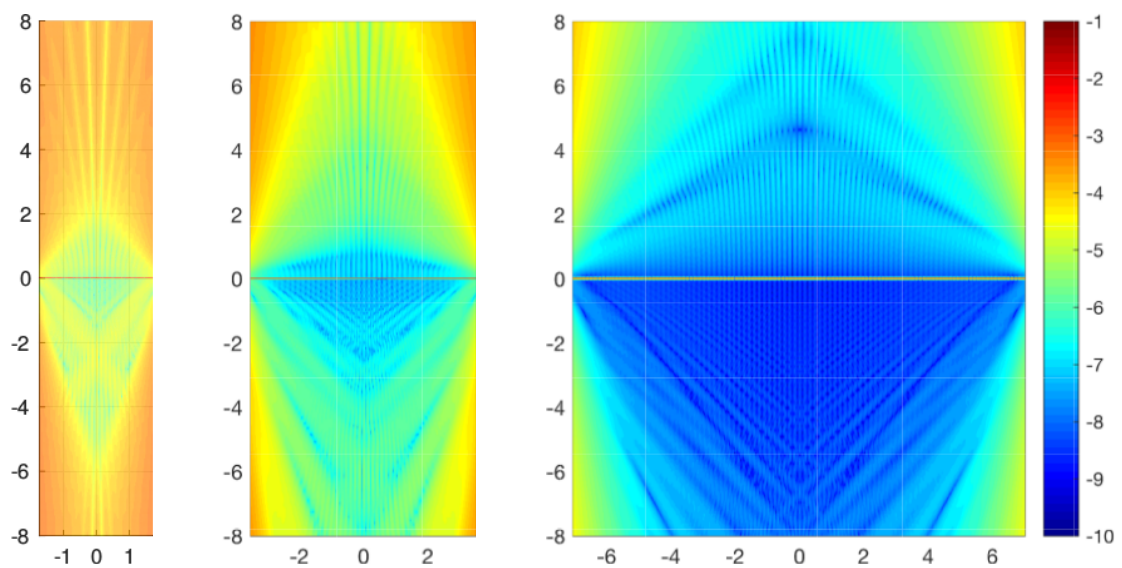

FIG. 4.2. Logarithm of the field errors $\log _{10}\left|u_{\xi}(\boldsymbol{x})-u_{\xi}^{w}(\boldsymbol{x})\right|$ obtained by means of the WGF method for the problem of scattering of $u_{\xi}^{\mathrm{inc}}, \xi=0$, defined in (4.1) for $\boldsymbol{y}=(0,1)$, by a dielectric plane for various window sizes $A=8 \lambda$ (left), $16 \lambda$ (middle), and $32 \lambda$ (right) and wavenumbers $k_{1}=20$ and $k_{2}=40\left(\lambda=2 \Pi / k_{1}\right)$. As in Figure $4.1, u_{\xi}^{w}$ is defined in (4.5), and $u_{\xi}$ equals the exact reflected field $C_{\xi}\left(T_{\xi}-1\right) \mathrm{e}^{i \xi x_{1}-\gamma_{1}(\xi) x_{2}}$ in the upper half-plane $\left\{x_{2}>0\right\}$ and the exact transmitted field $C_{\xi} T_{\xi} \mathrm{e}^{i \xi x_{1}+\gamma_{2}(\xi) x_{2}}$ in the lower half-plane $\left\{x_{2}<0\right\}$. The absolute errors on the segment $\left\{\left(x_{1}, x_{2}\right):-1 \leq x_{1} \leq 1\right.$ and $\left.x_{2}=4\right\}$, for example, are $10^{-3.68}, 10^{-5.18}$, and $10^{-7.04}$ for $A=8 \lambda$, $A=16 \lambda$, and $A=32 \lambda$, respectively, thus demonstrating superalgebraic convergence. Note that given the highly oscillatory character of the error, in both the horizontal direction and, at a much lower frequency, the vertical direction, it is difficult to obtain a clean (nonoscillatory) convergence pattern at any given point in space.

method in the solution of the problem of scattering of a point-source incident field (where the source is located at a point $\boldsymbol{y}=\left(y_{1}, y_{2}\right)$ with $y_{2}>0$ ) by a flat dielectric halfplane in the region $\left\{-c A \leq x_{1} \leq c A\right\}$. The resulting integral equation formulation for this problem is once again

$$
E \phi_{\boldsymbol{y}}+T_{\Pi}\left[\phi_{\boldsymbol{y}}\right]=\phi_{\boldsymbol{y}}^{\mathrm{inc}} \text { on } \Pi,
$$

where the right-hand side is now given by

$$
\boldsymbol{\phi}_{\boldsymbol{y}}^{\text {inc }}=\frac{i}{4}\left[\left.H_{0}^{(1)}\left(k_{1}|\cdot-\boldsymbol{y}|\right)\right|_{\Pi},\left.\frac{\partial}{\partial x_{2}} H_{0}^{(1)}\left(k_{1}|\cdot-\boldsymbol{y}|\right)\right|_{\Pi}\right]^{T} .
$$

According to well-known formula

$$
\frac{i}{4} H_{0}^{(1)}\left(k_{j}|\boldsymbol{x}-\boldsymbol{y}|\right)=\frac{1}{4 \pi} \int_{\mathcal{L}} \frac{\mathrm{e}^{-\gamma_{j}\left|x_{2}-y_{2}\right|}}{\gamma_{j}} \mathrm{e}^{i \xi\left(x_{1}-y_{1}\right)} \mathrm{d} \xi
$$

(where $\mathcal{L}=(-\infty, \infty)$ ), the field produced by a point source can be expressed as a superposition of incident fields $u_{\xi}^{\text {inc }}$ as defined in (4.1). But, to obtain a superposition which includes favorable plane-waves only (that is, plane-waves for which, like the ones considered in section 4.1 and above in the present section, the WGF method gives rise to superalgebraic convergence), we resort to Cauchy's theorem to deform the integration contour in the integral (4.12) so that the modified integration contour in the complex plane, denoted by $\mathcal{C}$ (see Figure 4.3), avoids the singular points $\xi= \pm k_{2}$ and $\xi= \pm k_{1}$ at which the WGF method fails. According to section 4.2, for each $\xi \in \mathcal{C}$ 


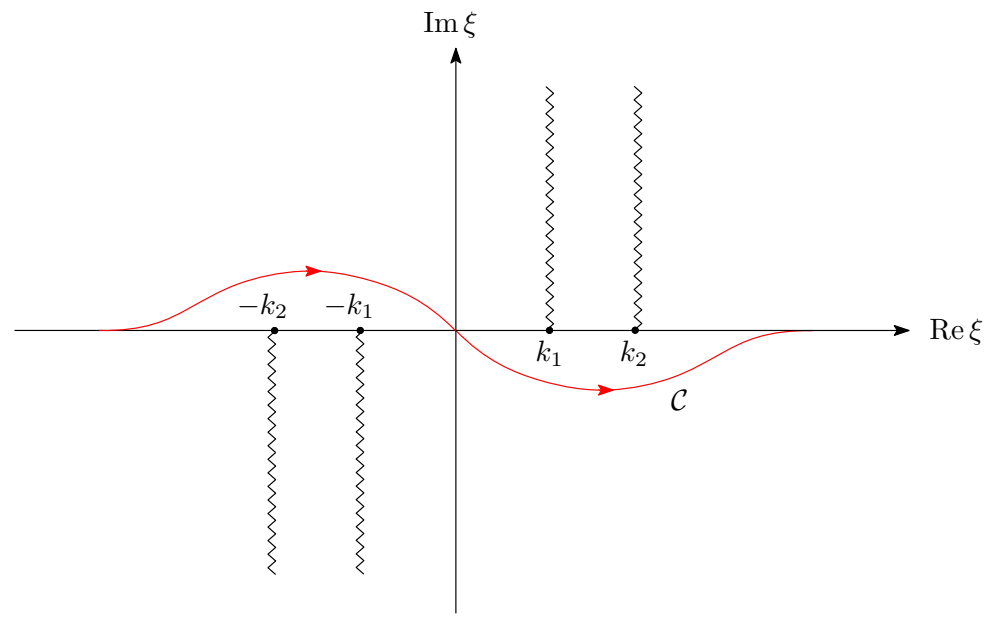

FIG. 4.3. Complex integration path utilized in the evaluation of the integral in (4.12).
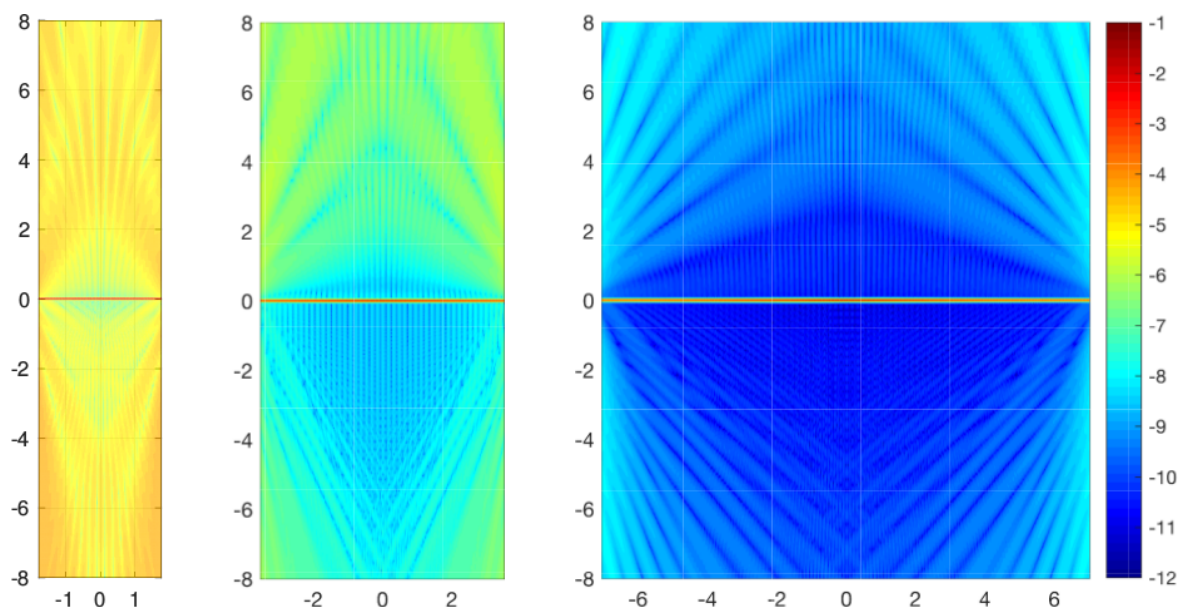

FIG. 4.4. Base-10 logarithm of the absolute error (see Remark 5.1) in the WGF-computed reflected and transmitted fields for the problem of scattering of a point-source incidence field by a dielectric plane.

the WGF method approximates, with superalgebraically small errors, the field resulting from the scattering of $u_{\xi}^{\text {inc }}$. Thus, in view of $(4.12)$ with $\mathcal{L}=\mathcal{C}$, we see that the solution of the integral equation (4.11) for point source illumination is also approximated with superalgebraically small errors throughout the interval $[-c A, c A]$-as illustrated in Figure 4.4.

Remark 4.2. The approximation properties demonstrated above in this section for incident fields given by point sources can easily be extended to illuminations given by surface distributions of point sources of the form

$$
\tilde{u}^{\text {inc }}(\boldsymbol{x})=\frac{i}{4} \int_{S} H_{0}^{(1)}\left(k_{1}|\boldsymbol{x}-\boldsymbol{y}|\right) \sigma(\boldsymbol{y}) \mathrm{d} s_{\boldsymbol{y}},
$$

where $S$ is a bounded curve contained in the upper half-plane $\left\{x_{2}>0\right\}$ (Figure 4.5) and $\sigma$ denotes a given surface density. Indeed, letting $\phi^{\text {inc }}=\left[\left.\tilde{u}^{\text {inc }}\right|_{\Pi}, \partial \tilde{u}^{\text {inc }} /\left.\partial n\right|_{\Pi}\right]^{T}$, 
the solutions $\phi=\phi(\boldsymbol{x})$ and $\phi^{w}=\phi^{w}(\boldsymbol{x})$ of the exact and windowed integral equations

$$
E \phi+T_{\Pi}[\phi]=\phi^{\text {inc }} \quad \text { on } \quad \Pi
$$

and

$$
E \phi^{w}+T_{\Pi}\left[W_{A} \phi^{w}\right]=\phi^{\text {inc }} \quad \text { on } \quad \Pi_{A}
$$

are given by integrals of the form

$$
\int_{S} \boldsymbol{\eta}_{\boldsymbol{y}}(\boldsymbol{x}) \sigma(\boldsymbol{y}) \mathrm{d} s_{\boldsymbol{y}}
$$

where $\boldsymbol{\eta}_{\boldsymbol{y}}=\boldsymbol{\phi}_{\boldsymbol{y}}$ (resp., $\boldsymbol{\eta}_{\boldsymbol{y}}=\boldsymbol{\phi}_{\boldsymbol{y}}^{w}$ ) is the exact solution (resp., the WGF approximation of the solution) of (4.11). Since, in view of the discussion presented above in this section, $\phi_{\boldsymbol{y}}^{w}$ is a superalgebraically uniformly accurate approximation of the corresponding solution $\boldsymbol{\phi}_{\boldsymbol{y}}$ throughout the region $[-c A, c A]$ for all point sources $\boldsymbol{y} \in S$, it follows that $\phi^{w}$ must itself be a superalgebraically accurate approximation of $\phi$ within the region $[-c A, c A]$. Similarly, the use of the representation formula (4.5) with densities $\phi^{w}$ produces the associated reflected and transmitted fields with superalgebraically small errors within the strip $[-c A, c A] \times \mathbb{R}$ and, in particular, throughout the curve $S$. An entirely analogous discussion applies, finally, to illumination by incident fields given by dipole distributions of the form

$$
\tilde{u}^{\text {inc }}(\boldsymbol{x})=\frac{i}{4} \int_{S} \frac{\partial}{\partial n_{\boldsymbol{y}}} H_{0}^{(1)}\left(k_{1}|\boldsymbol{x}-\boldsymbol{y}|\right) \sigma(\boldsymbol{y}) \mathrm{d} s_{\boldsymbol{y}}
$$

The contents of this remark play an important role in the multiple-scattering error analysis presented in section 4.4 .

4.4. Formal error analysis via multiple scattering. To place the descriptive discussions in section 3 within a more mathematically precise framework, this section presents a formal error analysis based on multiple-scattering iterations. For clarity and simplicity we limit the discussion in this section to geometrical configurations in which a defect, in the form of a dielectric obstacle bounded by the curve $S$, lies completely above the dielectric planar interface $\Pi$-as depicted in Figure 4.5.

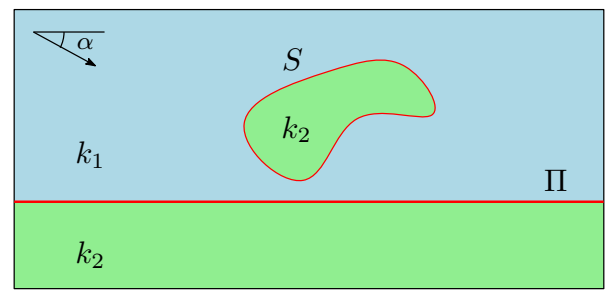

FIG. 4.5. Description of the domain consisting of an obstacle above a dielectric half-plane utilized in the multiple-scattering discussion in section 4.4. For notational simplicity the wavenumber within $S$ was selected to equal the "ground" wavenumber $k_{2}$. But this is otherwise an absolutely unessential assumption.

The specifics in the context of the configuration in Figure 4.5 are as follows. Upon illumination of such a structure by a plane-wave $u^{\text {inc }}(\boldsymbol{x})=\mathrm{e}^{i k_{1}\left(x_{1} \cos \alpha+x_{2} \sin \alpha\right)}$ 
and letting $\phi_{\Pi}^{\text {inc }}=\left[\left.u^{\text {inc }}\right|_{\Pi}, \partial u^{\text {inc }} /\left.\partial n\right|_{\Pi}\right]^{T}, \phi_{S}^{\text {inc }}=\left[\left.u^{\text {inc }}\right|_{S}, \partial u^{\text {inc }} /\left.\partial n\right|_{S}\right]^{T}$, the integral equations (2.6) may be re-expressed as the equation system

$$
\begin{aligned}
& E \phi_{\Pi}+T_{\Pi}\left[\phi_{\Pi}\right]=\phi_{\Pi}^{\text {inc }}+R_{S}^{\Pi}\left[\phi_{S}\right] \text { on } \Pi \text {, } \\
& E \phi_{S}+T_{S}\left[\phi_{S}\right]=\phi_{S}^{\text {inc }}+R_{\Pi}^{S}\left[\phi_{\Pi}\right] \quad \text { on } S
\end{aligned}
$$

for the unknowns $\phi_{S}=\left[\left.u_{2}\right|_{S}, \partial u_{2} /\left.\partial n\right|_{S}\right]^{T}$ and $\phi_{\Pi}=\left[\left.u_{2}\right|_{\Pi}, \partial u_{2} /\left.\partial n\right|_{\Pi}\right]^{T}$ (whose components are the values of the total field and its normal derivative at the boundaries $S$ and $\Pi$, respectively). Here the operators $T_{\Pi}$ and $T_{S}$ are defined as in (2.7)-(2.8) with $\Gamma=\Pi$ and $\Gamma=S$, respectively, and the operators $R_{S}^{\Pi}$ and $R_{\Pi}^{S}$ are given by

$$
\begin{aligned}
& R_{S}^{\Pi}[\phi](\boldsymbol{x})=\left[\begin{array}{c}
\int_{S}\left\{\frac{\partial G_{1}}{\partial n_{\boldsymbol{y}}}(\boldsymbol{x}, \boldsymbol{y}) \phi_{1}(\boldsymbol{y})-\nu G_{1}(\boldsymbol{x}, \boldsymbol{y}) \phi_{2}(\boldsymbol{y})\right\} \mathrm{d} s_{\boldsymbol{y}} \\
\int_{S}\left\{\frac{\partial^{2} G_{1}}{\partial n_{\boldsymbol{x}} \partial n_{\boldsymbol{y}}}(\boldsymbol{x}, \boldsymbol{y}) \phi_{1}(\boldsymbol{y})-\nu \frac{\partial G_{1}}{\partial n_{\boldsymbol{x}}}(\boldsymbol{x}, \boldsymbol{y}) \phi_{2}(\boldsymbol{y})\right\} \mathrm{d} s_{\boldsymbol{y}}
\end{array}\right], \boldsymbol{x} \in \Pi, \\
& R_{\Pi}^{S}[\boldsymbol{\phi}](\boldsymbol{x})=\left[\begin{array}{c}
\int_{\Pi}\left\{\frac{\partial G_{1}}{\partial n_{\boldsymbol{y}}}(\boldsymbol{x}, \boldsymbol{y}) \phi_{1}(\boldsymbol{y})-\nu G_{1}(\boldsymbol{x}, \boldsymbol{y}) \phi_{2}(\boldsymbol{y})\right\} \mathrm{d} s_{\boldsymbol{y}} \\
\int_{\Pi}\left\{\frac{\partial^{2} G_{1}}{\partial n_{\boldsymbol{x}} \partial n_{\boldsymbol{y}}}(\boldsymbol{x}, \boldsymbol{y}) \phi_{1}(\boldsymbol{y})-\nu \frac{\partial G_{1}}{\partial n_{\boldsymbol{x}}}(\boldsymbol{x}, \boldsymbol{y}) \phi_{2}(\boldsymbol{y})\right\} \mathrm{d} s_{\boldsymbol{y}}
\end{array}\right], \boldsymbol{x} \in S .
\end{aligned}
$$

In order to estimate the errors that arise as the system (4.17) is solved by means of the WGF method, we reformulate (4.17) in terms of the new unknowns $\widetilde{\phi}_{\Pi}=\phi_{\Pi}-\phi_{\Pi}^{f}$ on $\Pi$ and $\widetilde{\phi}_{S}=\phi_{S}-\phi_{S}^{f}$ on $S$. Here, denoting by $u_{2}^{f}$ and $u^{f}$ the exact transmitted field and the total field for the obstacle-free problem, respectively (see (A.1) in Appendix A of the supplementary materials), we have set $\phi_{\Pi}^{f}=\left[\left.u_{2}^{f}\right|_{\Pi}, \partial u_{2}^{f} /\left.\partial n\right|_{\Pi}\right]^{T}$ and $\phi_{S}^{f}=$ $\left[\left.u^{f}\right|_{S}, \partial u^{f} /\left.\partial n\right|_{S}\right]^{T}$. Using the identities $E \phi_{\Pi}^{f}+T_{\Pi}\left[\boldsymbol{\phi}_{\Pi}^{f}\right]=\boldsymbol{\phi}_{\Pi}^{\text {inc }}$ on $\Pi, R_{S}^{\Pi}\left[\boldsymbol{\phi}_{S}^{f}\right]=\mathbf{0}$, and $R_{\Pi}^{S}\left[\phi_{\Pi}^{f}\right]=\phi_{S}^{f}-\phi_{S}^{\text {inc }}$, which follow from Green's theorem (using (A.12) and (A.2) in Appendix A of the supplementary materials) and replacing $\phi_{\Pi}=\widetilde{\phi}_{\Pi}+\phi_{\Pi}^{f}$ and $\phi_{S}=\widetilde{\phi}_{S}+\phi_{S}^{f}$ in $(4.17 \mathrm{a})$ and $(4.17 \mathrm{~b})$, respectively, we obtain the following integral equation system:

$$
\begin{aligned}
E \widetilde{\boldsymbol{\phi}}_{\Pi}+T_{\Pi}\left[\widetilde{\boldsymbol{\phi}}_{\Pi}\right] & =R_{S}^{\Pi}\left[\widetilde{\boldsymbol{\phi}}_{S}\right] \quad \text { on } \Pi, \\
E \widetilde{\boldsymbol{\phi}}_{S}+T_{S}\left[\widetilde{\boldsymbol{\phi}}_{S}\right] & =\phi_{S}^{f}+R_{\Pi}^{S}\left[\widetilde{\phi}_{\Pi}\right] \text { on } S .
\end{aligned}
$$

The multiple-scattering character of the problem embodied in (4.20) (or, equivalently, (4.17)) can be elucidated by means of the formal Neumann series solution

$$
\left[\begin{array}{c}
\widetilde{\boldsymbol{\phi}}_{\Pi} \\
\widetilde{\boldsymbol{\phi}}_{S}
\end{array}\right]=\sum_{n=0}^{\infty}\left[\begin{array}{c}
\tilde{\boldsymbol{\phi}}_{\Pi}^{(n)} \\
\tilde{\boldsymbol{\phi}}_{S}^{(n)}
\end{array}\right]=\sum_{n=0}^{\infty}\left[\begin{array}{cc}
0 & \left(E+T_{\Pi}\right)^{-1} R_{S}^{\Pi} \\
\left(E+T_{S}\right)^{-1} R_{\Pi}^{S} & 0
\end{array}\right]^{n}\left[\begin{array}{c}
\mathbf{0} \\
\left(E+T_{S}\right)^{-1} \boldsymbol{\phi}_{S}^{f}
\end{array}\right] .
$$

Clearly $\tilde{\boldsymbol{\phi}}_{\Pi}^{(0)}=\mathbf{0}$, and $\boldsymbol{\phi}_{S}^{(0)}$ is the solution of the integral equation

$$
E \tilde{\phi}_{S}^{(0)}+T_{S}\left[\tilde{\phi}_{S}^{(0)}\right]=\phi_{S}^{f} \quad \text { on } \quad S .
$$

The $n>0$ terms in the series, in turn, satisfy the recurrence relation

$$
\left[\begin{array}{c}
\tilde{\phi}_{\Pi}^{(n)} \\
\tilde{\phi}_{S}^{(n)}
\end{array}\right]=\left[\begin{array}{cc}
\left(E+T_{\Pi}\right)^{-1} & 0 \\
0 & \left(E+T_{S}\right)^{-1}
\end{array}\right]\left[\begin{array}{cc}
0 & R_{\Pi}^{S} \\
R_{S}^{\Pi} & 0
\end{array}\right]\left[\begin{array}{c}
\tilde{\phi}_{\Pi}^{(n-1)} \\
\phi_{S}^{(n-1)}
\end{array}\right],
$$

Copyright (C) by SIAM. Unauthorized reproduction of this article is prohibited. 
or, equivalently,

$$
\begin{aligned}
E \tilde{\boldsymbol{\phi}}_{\Pi}^{(n)}+T_{\Pi}\left[\tilde{\boldsymbol{\phi}}_{\Pi}^{(n)}\right] & =R_{S}^{\Pi}\left[\tilde{\boldsymbol{\phi}}_{S}^{(n-1)}\right] \quad \text { on } \quad \Pi, \\
E \tilde{\boldsymbol{\phi}}_{S}^{(n)}+T_{S}\left[\tilde{\boldsymbol{\phi}}_{S}^{(n)}\right] & =R_{\Pi}^{S}\left[\tilde{\boldsymbol{\phi}}_{\Pi}^{(n-1)}\right] \quad \text { on } \quad S .
\end{aligned}
$$

The corresponding "multiple-scattering" form of the windowed integral equations (3.4) for the configuration depicted in Figure 4.5, in turn, is given by the system

$$
\begin{aligned}
E \boldsymbol{\psi}_{\Pi}+T_{\Pi}\left[W_{A} \boldsymbol{\psi}_{\Pi}\right] & =\boldsymbol{\phi}_{\Pi}^{\mathrm{inc}}-T_{\Pi}\left[\left(I-W_{A}\right) \boldsymbol{\phi}_{\Pi}^{f}\right]+R_{S}^{\Pi}\left[\boldsymbol{\psi}_{S}\right] \quad \text { on } \quad \Pi, \\
E \boldsymbol{\psi}_{S}+T_{S}\left[\boldsymbol{\psi}_{S}\right] & =\boldsymbol{\phi}_{S}^{\mathrm{inc}}+R_{\Pi}^{S}\left[W_{A} \boldsymbol{\psi}_{\Pi}\right]+R_{\Pi}^{S}\left[\left(I-W_{A}\right) \boldsymbol{\phi}_{\Pi}^{f}\right] \quad \text { on } \quad S,
\end{aligned}
$$

which, letting $\widetilde{\boldsymbol{\psi}}_{\Pi}=\boldsymbol{\psi}_{\Pi}-\boldsymbol{\phi}_{\Pi}^{f}$ on $\Pi_{A}$ and $\widetilde{\boldsymbol{\psi}}_{S}=\boldsymbol{\psi}_{S}-\boldsymbol{\phi}_{S}^{f}$ on $S$, becomes

$$
\begin{aligned}
E \widetilde{\boldsymbol{\psi}}_{\Pi}+T_{\Pi}\left[W_{A} \widetilde{\boldsymbol{\psi}}_{\Pi}\right] & =R_{S}^{\Pi}\left[\widetilde{\boldsymbol{\psi}}_{S}\right] \quad \text { on } \Pi_{A}, \\
E \widetilde{\boldsymbol{\psi}}_{S}+T_{S}\left[\widetilde{\boldsymbol{\psi}}_{S}\right] & =\boldsymbol{\phi}_{S}^{f}+R_{\Pi}^{S}\left[W_{A} \widetilde{\boldsymbol{\psi}}_{\Pi}\right] \text { on } S,
\end{aligned}
$$

where $\Pi_{A}=\Pi \cap[-A, A]$. Thus, comparison with (4.20) shows that, similarly, the multiple-scattering recursion for the windowed problem is initialized by $\tilde{\boldsymbol{\psi}}_{\Pi}^{(0)}=\mathbf{0}$ on $\Pi_{A}$ and

$$
E \tilde{\boldsymbol{\psi}}_{S}^{(0)}+T_{S}\left[\tilde{\boldsymbol{\psi}}_{S}^{(0)}\right]=\boldsymbol{\phi}_{S}^{f} \quad \text { on } \quad S,
$$

with $n>0$ terms given by the solutions of the equations

$$
\begin{aligned}
E \tilde{\boldsymbol{\psi}}_{\Pi}^{(n)}+T_{\Pi}\left[W_{A} \tilde{\boldsymbol{\psi}}_{\Pi}^{(n)}\right] & =R_{S}^{\Pi}\left[\tilde{\boldsymbol{\psi}}_{S}^{(n-1)}\right] \quad \text { on } \Pi_{A}, \\
E \tilde{\boldsymbol{\psi}}_{S}^{(n)}+T_{S}\left[\tilde{\boldsymbol{\psi}}_{S}^{(n)}\right] & =R_{\Pi}^{S}\left[W_{A} \tilde{\boldsymbol{\psi}}_{\Pi}^{(n-1)}\right] \quad \text { on } S .
\end{aligned}
$$

Since (4.21) and (4.24) coincide, so do their solutions: the $n=0$ approximation produced by the WGF method, which is given by $\tilde{\boldsymbol{\psi}}_{S}^{(0)}$, coincides with the exact solution $\tilde{\phi}_{S}^{(0)}$ throughout $S$. Similarly, $\tilde{\boldsymbol{\psi}}_{\Pi}^{(0)}=\mathbf{0}$ coincides with the restriction of $\tilde{\boldsymbol{\phi}}_{\Pi}^{(0)}=\mathbf{0}$ to $\Pi_{A}$. And, the same is true about the $n=1$ approximation on $S: \tilde{\boldsymbol{\psi}}_{S}^{(1)}=$ $\tilde{\phi}_{S}^{(1)}=\mathbf{0}$. But the $n=1$ approximation on $\Pi$ as well as all subsequent approximations do not coincide on either $\Pi$ or $S$. As shown in what follows, however, the WGF iterates $\tilde{\boldsymbol{\psi}}_{S}^{(n)}$ approximate the exact iterates $\tilde{\boldsymbol{\phi}}_{S}^{(n)}$ with superalgebraic accuracy.

In order to establish this approximation result (and thus complete our multiplescattering error analysis) we rely on the fact that the right-hand sides in (4.22a), (4.25a), (4.22b), and (4.25b) can be interpreted as scattered fields by either $S$ or $\Pi$ resulting from illumination by fields scattered in previous stages of the multi-scattering recurrence. For example, the right-hand side in (4.22a) (resp., (4.25a)) coincides with the values on $\Pi$ (resp., $\Pi_{A}$ ) of the fields scattered by $S$ under illumination given by $R_{\Pi}^{S}\left[\tilde{\phi}_{\Pi}^{(n-2)}\right]$ (resp., $R_{\Pi}^{S}\left[W_{A} \tilde{\psi}_{\Pi}^{(n-2)}\right]$ ). Similarly, the right-hand side in $(4.22 \mathrm{~b}$ ) (resp., (4.25b)) coincides with the values on $S$ of the fields scattered by $\Pi$ (resp., $\Pi_{A}$ ) under illumination given by $R_{S}^{\Pi}\left[\tilde{\boldsymbol{\phi}}_{S}^{(n-2)}\right]$ (resp., $R_{S}^{\Pi}\left[\tilde{\boldsymbol{\psi}}_{S}^{(n-2)}\right]$ ). 
On the strength of this observation, then, let us consider once again the $n=1$ terms $\tilde{\boldsymbol{\phi}}_{\Pi}^{(1)}$ and $\tilde{\boldsymbol{\psi}}_{\Pi}^{(1)}$ (on $\Pi_{A}$ ) which, as indicated above, do not exactly coincide. Noting that the incident fields in (4.22a) and (4.25a) are given by identical distributions of point sources along $S$, however, Remark 4.2 tells us that the WGF solution $\tilde{\boldsymbol{\psi}}_{\Pi}^{(1)}$ approximates $\tilde{\phi}_{\Pi}^{(1)}$ with superalgebraically small errors.

Continuing with the multiple-scattering process let us now consider the $n=2$ instance of (4.22b) and (4.25b). Relying once again on Remark 4.2, the established superalgebraic convergence of $\tilde{\boldsymbol{\psi}}_{\Pi}^{(1)}$ to $\tilde{\boldsymbol{\phi}}_{\Pi}^{(1)}$ throughout $[-c A, c A]$ implies, in turn, that $R_{\Pi}^{S}\left[W_{A} \tilde{\boldsymbol{\psi}}_{\Pi}^{(1)}\right]$ approximates $R_{\Pi}^{S}\left[\tilde{\phi}_{\Pi}^{(1)}\right]$ with superalgebraically small errors as well, and thus the stability of the integral equation posed on $S$ permits us to conclude that $\tilde{\boldsymbol{\psi}}_{S}^{(2)}$ approximates $\tilde{\phi}_{S}^{(2)}$ with superalgebraically small errors. In view of (4.22a) and (4.25a), on the other hand, we note that $\tilde{\boldsymbol{\phi}}_{\Pi}^{(2)}=\mathbf{0}$ on $\Pi$ and $\tilde{\boldsymbol{\psi}}_{\Pi}^{(2)}=\mathbf{0}$ on $\Pi_{A}$, as both equations have null right-hand sides.

Clearly, this argument can be carried out to all orders in perturbation theory, allowing us to conclude, within this formal framework, that, at least for the configuration depicted in Figure 4.5, the overall WGF method produces scattering solutions with superalgebraically small errors over the strip $[-c A, c A] \times \mathbb{R}$ (but see Remark 4.1). As indicated in sections 3.5 and 3.6, once such solutions are available, equally accurate solutions can easily be obtained over prescribed regions in space as well as in the far-field regions.

5. Numerical experiments. This section illustrates the proposed methodology with a variety of numerical results concerning dielectric media, including relevant efficiency and accuracy studies as well as generic application examples. For the sake of definiteness, test cases were only considered for dielectric materials (real $k_{2}$ values), but similar performance was observed for absorptive materials (complex $k_{2}$ values)including values for materials with low (resp., high) conductivity, such as limestone and saturated sand (resp., silt and clay).

Remark 5.1. In most cases considered in this paper, errors are reported as "relative errors in the $L^{\infty}$ norm," or just "relative errors," for short, but absolute $L^{\infty}$ errors are used as well. The "absolute error" over a given region is defined here as the maximum value of the error over every numerical grid point in that region. The relative error over a region, on the other hand, is defined as the quotient of the absolute error over the region by the maximum value of the solution over the region.

Our first example demonstrates the efficiency of the new approach by comparing the computing times required to create the systems of equations (which is the operation that dominates the computing time in all of the examples considered) for the WGF method (3.7) and the LGF method [26, equation (7)]. To do this we consider once again the configuration associated with Figure 3.4, i.e., the problem of scattering by a semicircular bump defect on a dielectric plane under TE-polarization. Figure 5.1 displays the computing times for various wavenumbers $k_{1}$ and $k_{2}=2 k_{1}$ for each method. The discretization density was held proportional to $k_{1}$ to properly resolve the oscillatory character of the integrands, and, in order to easily allow for pointwise comparison of the corresponding integral-density solutions, the same discretization was used for both methods on the semicircular bump. For each run the WGF parameters were optimized to produce $\phi^{w}$ with a relative error which did not exceed $5 \times 10^{-5}$ on the bump surface. Similarly, the key parameters in the implementation 


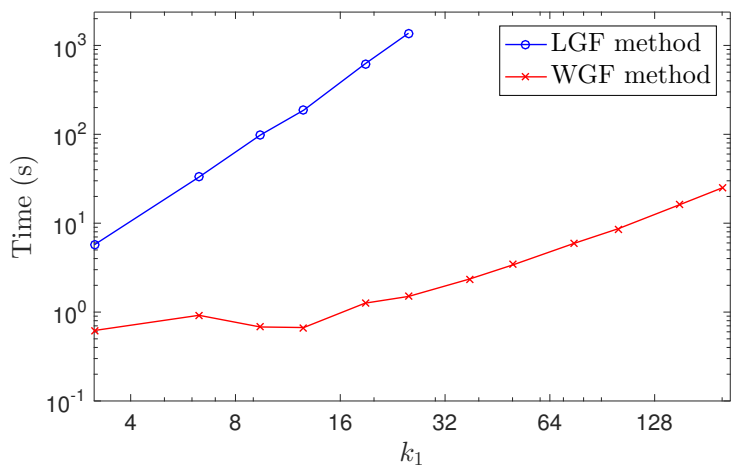

FIG. 5.1. Computing times required to form the linear systems of equations resulting from the Nyström discretization of the relevant integral equations for the WGF method (red line) and the LGF method [26] (blue line), as functions of the wavenumber $k_{1}$. The wavenumber $k_{2}$ was taken to equal $2 k_{1}$. Comparable ratios in computing times were obtained in cases in which complex $k_{2}$ values $\left(k_{2}=\omega \sqrt{\left(\varepsilon_{2}+i \sigma_{2} / \omega\right) \mu_{0}}\right)$ were assumed, with either large or small values of the conductivity $\sigma-$ including values for materials such as limestone, saturated sand, silt, and clay. Color is available online only.

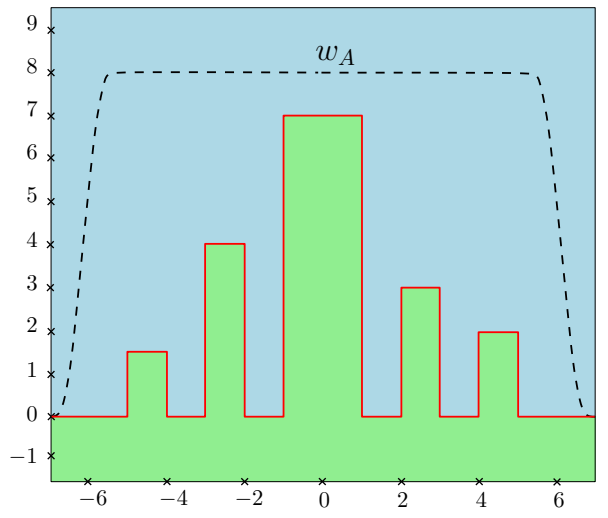

FIG. 5.2. City-like geometry and windowing function used. The windowing function $w_{A}$ was vertically stretched by a factor of 8 for visualization purposes.

of the LGF method (including the parameters associated with the numerical evaluation of the Sommerfeld integrals) were adjusted to yield the fastest computation of the corresponding integral density solution within a relative error of $5 \times 10^{-5}$. Note that the data points around $k_{1}=8 \pi \approx 25.1$ in Figure 5.1 (which is the last data point presented for the LGF method) shows that, for such frequencies, the WGF is approximately three orders of magnitude faster than the LGF method [26].

The problem of scattering by the city-like structure depicted in Figure 5.2 is considered next. Figure 5.2 also displays the window function utilized in this example. In contrast with the results presented previously in this paper, the case of TM-polarization was considered for this test. Table 5.1 reports the computing times required to form the relevant system matrices for both the WGF method and the LGF method. Both solvers were optimized to produce a absolute error of $5 \times 10^{-3}$ in the solutions of the integral equation, and the same computational grids were utilized 
to discretize the buildings for both methods.

Table 5.1 compares the computing times required by the WGF method and the LGF method for two values of $k_{2}$. In particular, we note not only that the new method is much faster than the previous approach, but also that the speed-up factor grows: a speed-up factor in the hundreds for the value $k_{2}=2 \pi$ is doubled as $k_{2}$ is itself doubled to the value $k_{2}=4 \pi$. Additionally, application of the LGF method in this context requires the use of fictitious curves underneath each building [26], each one of which (curves) itself must be discretized, while the WGF method requires discretization of the ground between the buildings and in the region where the windowing takes place. In the present case the LGF method produced a system of 2384 unknowns, while the WGF method produced a system of nearly identical size: 2406 unknowns. At higher frequencies, the WGF method requires fewer unknowns than the LGF method, since, as demonstrated in Table 5.2, at higher frequencies the width of the windowing function can be decreased while maintaining accuracy.

TABLE 5.1

Computing times required by the $L G F$ and WGF methods to produce integral equation solutions with an accuracy better than $5 \times 10^{-3}$ for the city-like geometry displayed in Figure 5.2 . We note that the LGF computing times for this problem are significantly larger than those considered in Figure 5.1 for similar wavenumbers. Such large costs arise in the present problem from the relatively large number of discretization points that need to be used near the plane $y=0$ to resolve the solution's corner singularity and from the high cost required by the associated Sommerfeld integral evaluation at such points.

\begin{tabular}{c|c|c|c|c}
\hline$k_{1}$ & $k_{2}$ & LGF time & WGF time & ratio \\
\hline$\pi$ & $2 \pi$ & $588 \mathrm{~s}$. & $3.07 \mathrm{~s}$. & 192 \\
\hline$\pi$ & $4 \pi$ & $3579 \mathrm{~s}$. & $9.10 \mathrm{~s}$. & 393 \\
\hline
\end{tabular}

TABLE 5.2

Extent of the windowed region required by the WGF method (3.7) to maintain an accuracy of $5 \times 10^{-5}$ in the approximation of the surface fields for the problem of scattering from a semicircular bump of unit radius with various wavenumbers. The angle of incidence was taken to equal $\alpha=-\pi / 8$.

\begin{tabular}{c|c|c}
\hline$k_{1}$ & $k_{2}$ & $A$ \\
\hline$\pi$ & $2 \pi$ & 6.5 \\
\hline $2 \pi$ & $4 \pi$ & 3.5 \\
\hline $4 \pi$ & $8 \pi$ & 1.75 \\
\hline $8 \pi$ & $16 \pi$ & 1.1875 \\
\hline
\end{tabular}

In our last numerical example we consider an obstacle over a rough ground which contains indentations below ground level. Figure 5.3 displays the geometry under consideration, together with a selection of window function (which yields an relative error of approximately $1 \%$ in the integral equation solution) and corresponding near fields under TE-polarized plane-wave illumination with incidence angle $\alpha=-\pi / 8$ and with $k_{1}=2 \pi$ and $k_{2}=4 \pi$. As demonstrated in Figure 5.4, superalgebraic convergence is once again observed as $A / \lambda$ grows. 


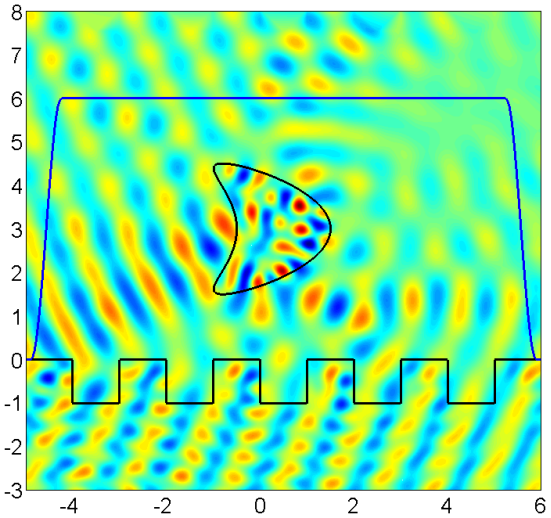

FIG. 5.3. Obstacle over a rough ground containing indentations below ground level and associated near fields. Interestingly, the rather narrow window function used (which was scaled vertically in this image for visual clarity) is wide enough to produce a relative error smaller than $1 \%$ in the integral equation solution.
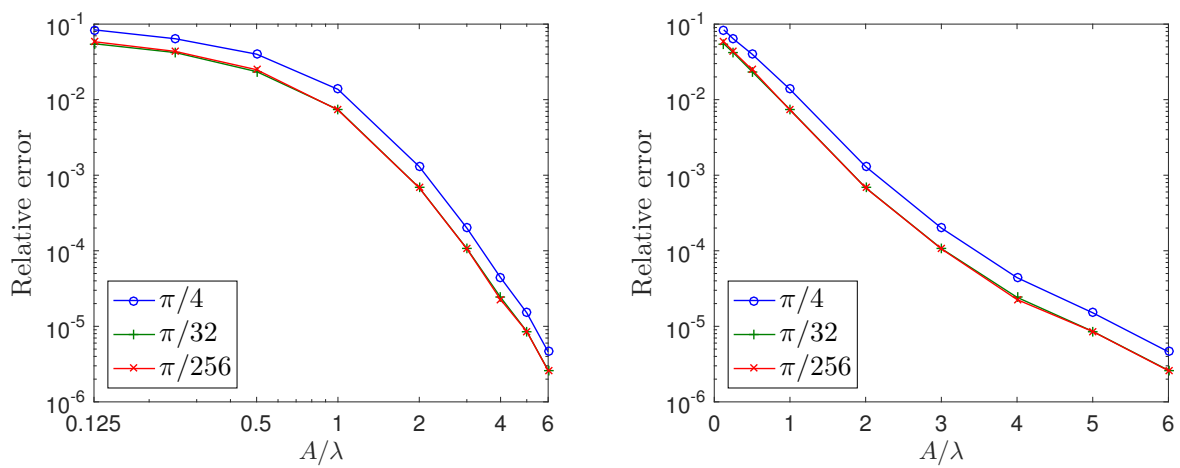

FIG. 5.4. Relative errors in the integral densities resulting from numerical solution of (3.7) for the structure depicted in Figure 5.3 by means of the full WGF method, for various window sizes and angles of incidence-including extremely shallow incidences. Left: log-log scale. Right: semi-log scale. Once again we see that the WGF method computes integral densities with superalgebraically high accuracy uniformly for all angles of incidence.

\section{REFERENCES}

[1] M. I. Aksun, A. Alparslan, and K. A. Michalski, Current status of closed-form Green's functions in layered media composed of natural and artificial materials, in Proceedings of the 2009 International Conference on Electromagnetics in Advanced Applications, 2009.

[2] A. Asheim and D. Huybrechs, Asymptotic analysis of numerical steepest descent with path approximations, Found. Comput. Math., 10 (2010), pp. 647-671.

[3] N. Bleistein, Mathematical Methods for Wave Phenomena, Academic Press, Orlando, FL, 1984.

[4] N. Bleistein and R. A. Handelsman, Asymptotic Expansions of Integrals, Courier Corporation, North Chelmsford, MA, 1975.

[5] O. P. Bruno And B. Delourme, Rapidly convergent two-dimensional quasi-periodic Green function throughout the spectrum-including Wood anomalies, J. Comput. Phys., 262 (2014), pp. 262-290.

Copyright (c) by SIAM. Unauthorized reproduction of this article is prohibited. 
[6] O. P. Bruno, C. Geuzaine, J. Monro, and F. Reitich, Prescribed error tolerances within fixed computational times for scattering problems of arbitrarily high frequency: The convex case, Philos. Trans. R. Soc. Lond. Ser. A Math. Phys. Eng. Sci., 362 (2004), pp. 629-645.

[7] W. CAI, Algorithmic issues for electromagnetic scattering in layered media: Green's functions, current basis, and fast solver, Adv. Comput. Math., 16 (2002), pp. 157-174.

[8] W. CAI AND T. YU, Fast calculations of dyadic Green's functions for electromagnetic scattering in a multilayered medium, J. Comput. Phys., 165 (2000), pp. 1-21.

[9] W. C. CHEw, Waves and Fields in Inhomogeneous Media, IEEE Press Ser. Electromagn. Wave Theory 522, IEEE Press, New York, 1995.

[10] D. Colton And R. Kress, Integral Equation Methods in Scattering Theory, Pure Appl. Math. (N. Y.), John Wiley \& Sons, New York, 1983; reprinted, SIAM, Philadelphia, 2013.

[11] D. Colton And R. KRess, Inverse Acoustic and Electromagnetic Scattering Theory, Appl. Math. Sci. 93, Springer Science \& Business Media, New York, 2012.

[12] T. J. Cui AND W. C. CHEw, Efficient evaluation of Sommerfeld integrals for TM wave scattering by buried objects, J. Electromagnet. Wave, 12 (1998), pp. 607-657.

[13] T. J. Cui AND W. C. CHEw, Fast evaluation of Sommerfeld integrals for EM scattering and radiation by three-dimensional buried objects, IEEE Trans. Geosci. Remote Sens., 37 (1999), pp. 887-900.

[14] L. Demanet And L. Ying, Scattering in flatland: Efficient representations via wave atoms, Found. Comput. Math., 10 (2010), pp. 569-613.

[15] J. A. DeSanto and P. A. Martin, On the derivation of boundary integral equations for scattering by an infinite one-dimensional rough surface, J. Acoust. Soc. Am., 102 (1997), pp. $67-77$.

[16] L. Ehrenpreis, Fourier Transforms in Several Complex Variables, Wiley-Interscience, New York, 1970.

[17] R. Kittappa and R. E. Kleinman, Acoustic scattering by penetrable homogeneous objects, J. Math. Phys., 16 (1975), pp. 421-432.

[18] R. KRess, A Nyström method for boundary integral equations in domains with corners, Numer. Math., 58 (1990), pp. 145-161.

[19] N. N. Lebedev, Special Functions and Their Applications, Prentice-Hall, Englewood Cliffs, NJ, 1965.

[20] I. V. Lindell and E. Alanen, Exact image theory for the Sommerfeld half-space problem, part I: Vertical magnetic dipole, IEEE Tran. Antennas and Propagation, 32 (1984), pp. 126133.

[21] A. Meier and S. N. Chandler-Wilde, On the stability and convergence of the finite section method for integral equation formulations of rough surface scattering, Math. Methods Appl. Sci., 24 (2001), pp. 209-232.

[22] D. Miret, G. Soriano, And M. Saillard, Rigorous simulations of microwave scattering from finite conductivity two-dimensional sea surfaces at low grazing angles, IEEE Trans. Geosci. Remote Sens., 52 (2014), pp. 3150-3158.

[23] J. A. Monro, JR., A Super-algebraically Convergent, Windowing-Based Approach to the Evaluation of Scattering from Periodic Rough Surfaces, Ph.D. thesis, Caltech, Pasadena, 2008.

[24] M. O'Neil, L. Greengard, and A. Pataki, On the efficient representation of the half-space impedance Green's function for the Helmholtz equation, Wave Motion, 51 (2014), pp. 1-13.

[25] M. Paulus, P. Gay-Balmaz, and O. Martin, Accurate and efficient computation of the Green's tensor for stratified media, Phys. Rev. E (3), 62 (2000), pp. 5797-5807.

[26] C. PÉrez-Arancibia And O. P. Bruno, High-order integral equation methods for problems of scattering by bumps and cavities on half-planes, J. Opt. Soc. Amer. A, 31 (2014), pp. 17381746.

[27] M. Saillard And G. Soriano, Rough surface scattering at low-grazing incidence: A dedicated model, Radio Science, 46 (2011), RS0E13.

[28] A. Sommerfeld, Über die Ausbreitung der Wellen in der drahtlosen Telegraphie, Ann. Phys., 333 (1909), pp. 665-736.

[29] P. Spiga, G. Soriano, And M. Saillard, Scattering of electromagnetic waves from rough surfaces: A boundary integral method for low-grazing angles, IEEE Trans. Antennas and Propagation, 56 (2008), pp. 2043-2050.

[30] Z. Zhao, L. Li, J. Smith, and L. CARIN, Analysis of scattering from very large threedimensional rough surfaces using MLFMM and ray-based analyses, IEEE Antennas and Propagation Magazine, 47 (2005), pp. 20-30.

Copyright (c) by SIAM. Unauthorized reproduction of this article is prohibited. 